\title{
Negligible Cooperation: Contrasting the Maximal- and Average-Error Cases
}

\author{
Parham Noorzad, Member, IEEE, Michael Langberg, Senior Member, IEEE, \\ and Michelle Effros, Fellow, IEEE
}

\begin{abstract}
In communication networks, cooperative strategies are coding schemes where network nodes work together to improve performance metrics such as the total rate delivered across the network. This work studies encoder cooperation in the setting of a discrete multiple access channel (MAC) with two encoders and a single decoder. A network node, here called the cooperation facilitator (CF), that is connected to both encoders via rate-limited links, enables the cooperation strategy. Previous work by the authors presents two classes of MACs: (i) one class where the average-error sum-capacity has an infinite derivative in the limit where CF output link capacities approach zero, and (ii) a second class of MACs where the maximal-error sum-capacity is not continuous at the point where the output link capacities of the CF equal zero. This work contrasts the power of the $\mathrm{CF}$ in the maximal- and average-error cases, showing that a constant number of bits communicated over the CF output link can yield a positive gain in the maximal-error sum-capacity, while a far greater number of bits, even a number that grows sublinearly in the blocklength, can never yield a non-negligible gain in the average-error sum-capacity.
\end{abstract}

Index Terms-Capacity region continuity, cooperation facilitator, edge removal problem, maximal-error capacity region, multiple access channel.

\section{INTRODUCTION}

Interference is an important limiting factor in the capacities of many communication networks. One way to reduce interference is to enable network nodes to work together to coordinate their transmissions. Strategies that employ coordinated transmissions are called cooperation strategies.

Perhaps the simplest cooperation strategy is "scheduled time-sharing" (e.g., [3, Theorem 15.3.2]), where nodes avoid interference by taking turns transmitting. A popular alternative model is the "conferencing" cooperation model [4]; in conferencing, unlike in time-sharing, encoders share information about the messages they wish to transmit and use that shared information to coordinate their channel inputs. In this work, we employ a similar approach, but in our cooperation model, encoders communicate indirectly. Specifically, the encoders communicate through another node, which we call the cooperation facilitator (CF) [5], [6]. Figure 1

This material is based upon work supported by the National Science Foundation under Grant Numbers 1527524 and 1526771, and has appeared in part in [1], [2].

P. Noorzad was with the California Institute of Technology, Pasadena, CA 91125 USA. (email: parham.n@outlook.com).

M. Langberg is with the State University of New York at Buffalo, Buffalo, NY 14260 USA (email: mikel@buffalo.edu).

M. Effros is with the California Institute of Technology, Pasadena, CA 91125 USA (email: effros@caltech.edu).

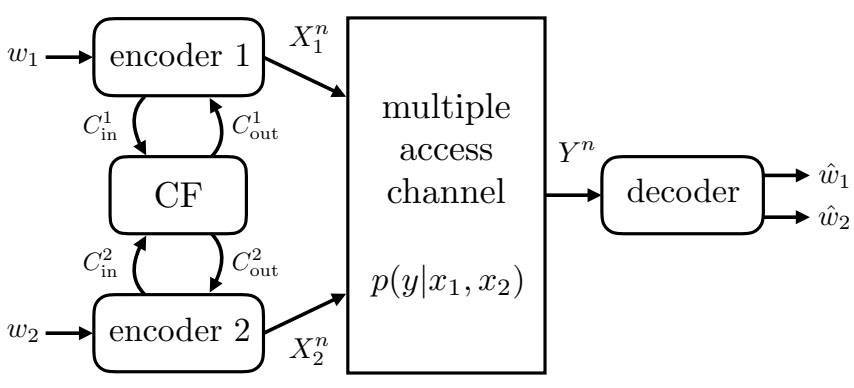

Fig. 1: A network consisting of two encoders that initially communicate via a $\mathrm{CF}$ and then using the information they receive, transmit their codewords over the MAC to the decoder.

depicts the CF model in the two-user multiple access channel (MAC) scenario.

The CF enables cooperation between the encoders through its rate-limited input and output links. Prior to choosing a codeword to transmit over the channel, each encoder sends a rate-limited function of its message to the CF. The CF uses the information it receives from both encoders to compute a rate-limited function for each encoder. It then transmits the computed values over its output links. Finally, each encoder selects a codeword using its message and the information it receives from the $\mathrm{CF}$.

To simplify our discussion in this section, suppose the CF input link capacities both equal $C_{\mathrm{in}}$ and the $\mathrm{CF}$ output link capacities both equal $C_{\text {out }}$. If $C_{\text {in }} \leq C_{\text {out }}$, then the optimal strategy for the $\mathrm{CF}$ is to simply forward the information it receives from one encoder to the other. Using the capacity region of the MAC with conferencing encoders [4], it follows that the average-error sum-capacity gain of CF cooperation in this case is bounded from above by $2 C_{\mathrm{in}}$ and does not depend on the precise value of $C_{\text {out }} \geq C_{\text {in }}$. If $C_{\mathrm{in}}>C_{\text {out }}$, however, the situation is more complicated since the $\mathrm{CF}$ cannot forward all of its incoming information. While the $2 C_{\text {in }}$ upper bound still holds, the dependence of the sum-capacity gain on $C_{\text {out }}$ is less clear. If the CF simply forwards part of the information it receives, then again by [4], the average-error sum-capacity gain is at most $2 C_{\text {out }}$. The $2 C_{\text {out }}$ bound has an intuitive interpretation: it reflects the amount of information the $\mathrm{CF}$ shares with the encoders, perhaps suggesting that the benefit of sharing information with rate $2 C_{\text {out }}$ with the encoders is at most $2 C_{\text {out }}$. It turns out, though, that a much larger gain is possible through more sophisticated coding techniques. 
Specifically, in prior work [6, Theorem 3], we show that there exist MACs such that for any fixed $C_{\text {in }}>0$, the averageerror sum-capacity has a derivative in $C_{\text {out }}$ that is infinite at $C_{\text {out }}=0$; that is, for small $C_{\text {out }}$, the gain resulting from cooperation exceeds any function that goes through the origin and has bounded derivative at that point.

The large sum-capacity gain described above is not limited to the average-error scenario. In fact, in related work [5. Proposition 5], we show that for any MAC for which, in the absence of cooperation, the average-error sum-capacity is strictly greater than the maximal-error sum-capacity, adding a $\mathrm{CF}$ and measuring the maximal-error sum-capacity for fixed $C_{\text {in }}>0$ gives a curve that is discontinuous at $C_{\text {out }}=0$. In this case, we say that "negligible cooperation" results in a non-negligible capacity benefit.

Given these earlier results, a number of important questions remain open in both the average- and maximal-error cases. For example, we wish to quantify the behavior of the average-error sum-capacity not only at the single point $C_{\text {out }}=0$, but rather for a range of $C_{\text {out }}$ values. We also seek to understand, in the average-error case, whether the sum-capacity gain can be discontinuous in $C_{\text {out }}$. In the maximal-error case, we would like to determine whether a constant number of $\mathrm{CF}$ output bits are sufficient to achieve the discontinuities already shown to be possible when the CF output link capacities tend to zero.

We next provide an overview of the key results in this work, which make progress towards addressing the above questions. Departing from the simplified discussion above, in our results we allow the $\mathrm{CF}$ input and output link capacities to differ. To this end, consider a discrete MAC with a $\left(\mathbf{C}_{\text {in }}, \mathbf{C}_{\text {out }}\right)$ CF where $\mathbf{C}_{\text {in }}:=\left(C_{\text {in }}^{1}, C_{\text {in }}^{2}\right)$ and $\mathbf{C}_{\text {out }}:=\left(C_{\text {out }}^{1}, C_{\text {out }}^{2}\right)$. To capture the dependence on these parameters, we denote the average- and maximal-error sum-capacities of this network with $C_{\text {sum }}\left(\mathbf{C}_{\text {in }}, \mathbf{C}_{\text {out }}\right)$ and $C_{\text {sum, max }}\left(\mathbf{C}_{\text {in }}, \mathbf{C}_{\text {out }}\right)$, respectively. Furthermore, let the components of $\mathbf{C}_{\mathrm{in}}^{*}=\left(C_{\mathrm{in}}^{* 1}, C_{\mathrm{in}}^{* 2}\right)$ be sufficiently large such that without loss of generality we can assume that for any $\mathbf{C}_{\text {out }} \in \mathbb{R}_{\geq 0}^{2}$, a $\left(\mathbf{C}_{\text {in }}^{*}, \mathbf{C}_{\text {out }}\right)$-CF has access to the messages of both encoders. Our results tackle both the special case where the $\mathrm{CF}$ has full knowledge of all the messages (i.e., $\mathbf{C}_{\mathrm{in}}=\mathbf{C}_{\mathrm{in}}^{*}$ ) and the general case where the $\mathrm{CF}$ may or may not have full knowledge (i.e., $\mathbf{C}_{\mathrm{in}}$ is arbitrary).

The CF with Full Knowledge: In this scenario, we explicitly observe the contrast between average- and maximalerror sum-capacities, showing that the former is continuous with respect to the $\mathrm{CF}$ output link capacities, while the latter is not only discontinuous, but also, a constant number of bits can suffice to achieve that discontinuity.

1) [Continuity of Average-Error Sum-Capacity in $\mathbf{C}_{\text {out }}$ ] Theorem 3 shows that for any discrete MAC, the mapping

$$
\mathbf{C}_{\text {out }} \mapsto C_{\text {sum }}\left(\mathbf{C}_{\text {in }}^{*}, \mathbf{C}_{\text {out }}\right)
$$

defined on $\mathbb{R}_{\geq 0}^{2}$ is continuous.

2) [Discontinuity of Maximal-Error Sum-Capacity in $\mathbf{C}_{\text {out }}$ ] Theorem 4 shows that there exists a discrete MAC where

$$
C_{\text {sum,max }}\left(\mathbf{C}_{\mathrm{in}}^{*}, k \text {-bit }\right)>C_{\text {sum, max }}\left(\mathbf{C}_{\mathrm{in}}^{*}, \mathbf{0}\right) .
$$

Here $C_{\text {sum,max }}\left(\mathbf{C}_{\text {in }}^{*}, k\right.$-bit $)$ denotes the maximal-error sum-capacity of a MAC and a CF that has full knowledge of the messages and sends at most $k$ bits back to the encoders over the entire blocklength.

The General CF: In this scenario, we can uniquely characterize the MACs that give a discontinuity in maximalerror sum-capacity. The average-error case, however, is more subtle. Our results for the average-error case include a lower bound on the sum-capacity gain. The upper bound, which is needed to establish continuity, proves to be more difficult and remains open in the general case.

1) [Square Root Lower Bound for Average-Error SumCapacity] Theorem 5 demonstrates that for a nonempty class of discrete MACs and any $\left(\mathbf{C}_{\text {in }}, \mathbf{C}_{\text {out }}\right) \in \mathbb{R}_{>0}^{2} \times$ $\mathbb{R}_{>0}^{2}$, the mapping

$$
h \mapsto C_{\text {sum }}\left(\mathbf{C}_{\text {in }}, h \mathbf{C}_{\text {out }}\right),
$$

grows at least as fast as $\sqrt{h}$ in the neighborhood of $h=$ $0^{+}$.

2) [Continuity of Average-Error Sum-Capacity in $\left.\left(\mathbf{C}_{\text {in }}, \mathbf{C}_{\text {out }}\right)\right]$ Theorem 6 provides sufficient conditions under which the average-error sum-capacity is continuous in the CF link capacities. Specifically, for any discrete MAC, $C_{\text {sum }}\left(\tilde{\mathbf{C}}_{\text {in }}, \tilde{\mathbf{C}}_{\text {out }}\right)$ is continuous at $\left(\tilde{\mathbf{C}}_{\text {in }}, \tilde{\mathbf{C}}_{\text {out }}\right)=\left(\mathbf{C}_{\text {in }}, \mathbf{C}_{\text {out }}\right)$ if any of the following conditions hold:

a) The $\mathrm{CF}$ and both encoders communicate: $C_{\mathrm{in}}^{i}>0$ and $C_{\text {out }}^{i}>0$ for $i \in\{1,2\}$.

b) The $\mathrm{CF}$ does not send information to either encoder: $C_{\text {out }}^{1}=0$ and $C_{\text {out }}^{2}=0$.

c) Both encoders send their full messages to the CF: $C_{\mathrm{in}}^{1} \geq C_{\mathrm{in}}^{1 *}$ and $C_{\mathrm{out}}^{2} \geq C_{\mathrm{out}}^{2 *}$.

d) At most one of the encoders sends its message to the CF: $C_{\text {in }}^{1}=0$ or $C_{\text {in }}^{2}=0$.

3) [Discontinuity of Maximal-Error Sum-Capacity in $\mathbf{C}_{\text {out }}$ : Necessary and Sufficient Condition] Theorem 8 states that for any discrete MAC and fixed $\mathbf{C}_{\mathrm{in}} \in \mathbb{R}_{>0}^{2}$, the mapping

$$
\mathbf{C}_{\text {out }} \mapsto C_{\text {sum,max }}\left(\mathbf{C}_{\text {in }}, \mathbf{C}_{\text {out }}\right)
$$

is not continuous if and only if

$$
C_{\text {sum }}(\mathbf{0}, \mathbf{0})>C_{\text {sum, } \max }(\mathbf{0}, \mathbf{0}) .
$$

The remainder of the paper is organized as follows. In Section II] we discuss related work from the literature. In Section III we present a precise description of our model. In Section IV], we review prior results on the sum-capacity gain of cooperation via a CF using the definitions from Section III. We state our contributions in Section V As some our results require lengthy proofs, prior to providing all the details in Section IX] we present outlines of the main arguments in Sections VI VII and VIII. We give a summary of this work in Section $\mathrm{X}$

\section{RELATED WORK}

A continuity problem similar to the one considered here appears in studying rate-limited feedback over the MAC. In that setting, Sarwate and Gastpar [7] use the dependencebalance bounds of Hekstra and Willems [8] to show that as 
the feedback rate converges to zero, the average-error capacity region converges to the average-error capacity region of the same MAC in the absence of feedback.

The problem we study here can also be formulated as an "edge removal problem" as introduced by Ho, Effros, and Jalali [9], [10]. The edge removal problem seeks to quantify the capacity effect of removing a single edge from a network. While bounds on this capacity impact exist in a number of limited scenarios (see, for example, [9] and [10]), the problem remains open in the general case. In the context of network coding, Langberg and Effros show that this problem is connected to a number of other open problems, including the difference between the 0 -error and $\epsilon$-error network coding capacity regions [11] and the difference between the lossless source coding regions for independent and dependent sources [12].

In [13], Kosut and Kliewer present different variations of the edge removal problem in a unified setting. In their terminology, one of the problems the present work investigates is whether the network consisting of a MAC and a CF satisfies the "weak edge removal property" with respect to the averageerror reliability criterion. A discussion in [14, Chapter 1] summarizes the known results for each variation of the edge removal problem as well.

The question of whether the capacity region of a network consisting of noiseless links is continuous with respect to the link capacities is studied by $\mathrm{Gu}$, Effros, and Bakshi [15] and Chan and Grant [16]. The present work differs from [15], [16] in the network under consideration; while our network does have noiseless links (the CF input and output links), it also contains a multiterminal component (the MAC) which may exhibit interference or noise; no such component appears in [15], [16].

For the maximal-error case, our study focuses on the effect of a constant number of bits of communication in the memoryless setting. For noisy networks with memory, it is not difficult to see that even one bit of communication may indeed affect the capacity region. For example, consider a binary symmetric channel whose error probability $\theta$ is chosen at random and then fixed for all time. If for $i \in\{1,2\}, \theta$ equals $\theta_{i}$ with positive probability $p_{i}$, and $0 \leq \theta_{1}<\theta_{2} \leq 1 / 2$, then a single bit of feedback (not rate 1 , but exactly one bit no matter how large the blocklength) from the receiver to the transmitter suffices to increase the capacity. For memoryless channels, the question is far more subtle and is the subject of our study.

In the next section, we present the cooperation model we consider in this work.

\section{The CoOperation FACILITATOR MOdEL}

In this work, we study cooperation between two encoders that communicate their messages to a decoder over a stationary, memoryless, and discrete MAC. Such a MAC can be represented by the triple

$$
\left(\mathcal{X}_{1} \times \mathcal{X}_{2}, p\left(y \mid x_{1}, x_{2}\right), \mathcal{Y}\right)
$$

where $\mathcal{X}_{1}, \mathcal{X}_{2}$, and $\mathcal{Y}$ are finite sets and $p\left(y \mid x_{1}, x_{2}\right)$ is a conditional probability mass function. For any positive integer $n \geq 2$, the $n$th extension of this MAC is given by

$$
p\left(y^{n} \mid x_{1}^{n}, x_{2}^{n}\right):=\prod_{t=1}^{n} p\left(y_{t} \mid x_{1 t}, x_{2 t}\right) .
$$

For each positive integer $n$, called the blocklength, and nonnegative real numbers $R_{1}$ and $R_{2}$, called the rates, we next define a $\left(2^{n R_{1}}, 2^{n R_{2}}, n\right)$-code for communication over a MAC with a $\left(\mathbf{C}_{\text {in }}, \mathbf{C}_{\text {out }}\right)-\mathrm{CF}$. Here $\mathbf{C}_{\text {in }}=\left(C_{\text {in }}^{1}, C_{\text {in }}^{2}\right)$ and $\mathbf{C}_{\text {out }}=\left(C_{\text {out }}^{1}, C_{\text {out }}^{2}\right)$ represent the capacities of the $\mathrm{CF}$ input and output links, respectively. (See Figure 1])

\section{A. Positive Rate Cooperation}

For every $x \geq 1$, let $[x]$ denote the set $\{1, \ldots,\lfloor x\rfloor\}$. For $i \in$ $\{1,2\}$, the transmission of encoder $i$ to the CF is represented by a mapping

$$
\varphi_{i}:\left[2^{n R_{i}}\right] \rightarrow\left[2^{n C_{\mathrm{in}}^{i}}\right] .
$$

The CF uses the information it receives from the encoders to compute a function

$$
\psi_{i}:\left[2^{n C_{\mathrm{in}}^{1}}\right] \times\left[2^{n C_{\mathrm{in}}^{2}}\right] \rightarrow\left[2^{n C_{\mathrm{out}}^{i}}\right]
$$

for encoder $i$, where $i \in\{1,2\}$. Encoder $i$ uses its message and what it receives from the $\mathrm{CF}$ to select a codeword according to

$$
f_{i}:\left[2^{n R_{i}}\right] \times\left[2^{n C_{\text {out }}^{i}}\right] \rightarrow \mathcal{X}_{i}^{n} .
$$

The decoder finds estimates of the transmitted messages using the channel output. It is represented by a mapping

$$
g: \mathcal{Y}^{n} \rightarrow\left[2^{n R_{1}}\right] \times\left[2^{n R_{2}}\right]
$$

The collection of mappings

$$
\left(\varphi_{1}, \varphi_{2}, \psi_{1}, \psi_{2}, f_{1}, f_{2}, g\right)
$$

defines a $\left(2^{n R_{1}}, 2^{n R_{2}}, n\right)$-code for the MAC with a $\left(\mathbf{C}_{\text {in }}, \mathbf{C}_{\text {out }}\right)-\mathrm{CF}$.

\section{B. Constant Size Cooperation}

To address the setting of a constant number of cooperation bits, we modify the output link of the CF to have support $\left[2^{k}\right]$ for some fixed integer $k$; unlike the prior support $\left[2^{n C_{\text {out }}^{i}}\right]$, the support of this link is independent of the blocklength $n$. Then, for $i \in\{1,2\}$, the transmission of encoder $i$ to the $\mathrm{CF}$ is represented by a mapping

$$
\varphi_{i}:\left[2^{n R_{i}}\right] \rightarrow\left[2^{n C_{\mathrm{in}}^{i}}\right]
$$

The $\mathrm{CF}$ uses the information it receives from the encoders to compute a function

$$
\psi_{i}:\left[2^{n C_{\mathrm{in}}^{1}}\right] \times\left[2^{n C_{\mathrm{in}}^{2}}\right] \rightarrow\left[2^{k}\right]
$$

for encoder $i$, where $i \in\{1,2\}$. Encoder $i$, as before, uses its message and what it receives from the CF to select a codeword according to

$$
f_{i}:\left[2^{n R_{i}}\right] \times\left[2^{k}\right] \rightarrow \mathcal{X}_{i}^{n} .
$$

We now say that

$$
\left(\varphi_{1}, \varphi_{2}, \psi_{1}, \psi_{2}, f_{1}, f_{2}, g\right)
$$

defines a $\left(2^{n R_{1}}, 2^{n R_{2}}, n\right)$-code for the MAC with a $\left(\mathbf{C}_{\text {in }}, k\right.$-bit)-CF. 


\section{Capacity Region}

For a fixed code, the probability of decoding a particular transmitted message pair $\left(w_{1}, w_{2}\right)$ incorrectly is given by

$\lambda_{n}\left(w_{1}, w_{2}\right):=\sum_{y^{n}: g\left(y^{n}\right) \neq\left(w_{1}, w_{2}\right)} p\left(y^{n} \mid f_{1}\left(w_{1}, z_{1}\right), f_{2}\left(w_{2}, z_{2}\right)\right)$,

where $z_{1}$ and $z_{2}$ are the CF outputs and are calculated, for $i \in\{1,2\}$, according to

$$
z_{i}=\psi_{i}\left(\varphi_{1}\left(w_{1}\right), \varphi_{2}\left(w_{2}\right)\right)
$$

The average probability of error is defined as

$$
P_{e, \text { avg }}^{(n)}:=\frac{1}{2^{n\left(R_{1}+R_{2}\right)}} \sum_{w_{1}, w_{2}} \lambda_{n}\left(w_{1}, w_{2}\right),
$$

and the maximal probability of error is given by

$$
P_{e, \max }^{(n)}:=\max _{w_{1}, w_{2}} \lambda_{n}\left(w_{1}, w_{2}\right) .
$$

A rate pair $\left(R_{1}, R_{2}\right)$ is achievable with respect to the average-error reliability criterion if there exists an infinite sequence of $\left(2^{n R_{1}}, 2^{n R_{2}}, n\right)$-codes such that $P_{e, \text { avg }}^{(n)} \rightarrow 0$ as $n \rightarrow \infty$. The average-error capacity region of a MAC with a $\left(\mathbf{C}_{\text {in }}, \mathbf{C}_{\text {out }}\right)-\mathrm{CF}$, denoted by $\mathscr{C}_{\text {avg }}\left(\mathbf{C}_{\text {in }}, \mathbf{C}_{\text {out }}\right)$, is the closure of the set of all rate pairs that are achievable with respect to the average-error reliability criterion. The average-error sumcapacity is defined as

$$
C_{\text {sum }}\left(\mathbf{C}_{\text {in }}, \mathbf{C}_{\text {out }}\right):=\max _{\left(R_{1}, R_{2}\right) \in \mathscr{C}_{\text {avg }}\left(\mathbf{C}_{\text {in }}, \mathbf{C}_{\text {out }}\right)}\left(R_{1}+R_{2}\right) .
$$

By replacing $P_{e, \text { avg }}^{(n)}$ with $P_{e, \text { max }}^{(n)}$, we can similarly define achievable rates with respect to the maximal-error reliability criterion, the maximal-error capacity region, and the maximalerror sum-capacity. For a MAC with a $\left(\mathbf{C}_{\text {in }}, \mathbf{C}_{\text {out }}\right)-\mathbf{C F}$, we denote the maximal-error capacity region and sum-capacity by $\mathscr{C}_{\text {max }}\left(\mathbf{C}_{\text {in }}, \mathbf{C}_{\text {out }}\right)$ and $C_{\text {sum,max }}\left(\mathbf{C}_{\text {in }}, \mathbf{C}_{\text {out }}\right)$, respectively.

\section{Prior Results on the Sum-Capacity Gain of COOPERATION}

We next review a number of results from [5], [6] which describe the sum-capacity gain of cooperation under the CF model. We begin with the average-error case.

Consider a discrete $\operatorname{MAC}\left(\mathcal{X}_{1} \times \mathcal{X}_{2}, p\left(y \mid x_{1}, x_{2}\right), \mathcal{Y}\right)$. Let $p_{\text {ind }}\left(x_{1}, x_{2}\right)=p_{\text {ind }}\left(x_{1}\right) p_{\text {ind }}\left(x_{2}\right)$ be a distribution that satisfies

$$
\begin{aligned}
I_{\text {ind }}\left(X_{1}, X_{2} ; Y\right) & :=\left.I\left(X_{1}, X_{2} ; Y\right)\right|_{p_{\text {ind }}\left(x_{1}, x_{2}\right)} \\
& =\max _{p\left(x_{1}\right) p\left(x_{2}\right)} I\left(X_{1}, X_{2} ; Y\right) ;
\end{aligned}
$$

subscript "ind" here denotes the independence of the codewords from encoders 1 and 2 in the absence of cooperation. Next, for an arbitrary distribution $p_{\text {dep }}\left(x_{1}, x_{2}\right)$, define

$$
I_{\mathrm{dep}}\left(X_{1}, X_{2} ; Y\right):=\left.I\left(X_{1}, X_{2} ; Y\right)\right|_{p_{\mathrm{dep}}\left(x_{1}, x_{2}\right)} .
$$

Furthermore, let $p_{\text {ind }}(y)$ and $p_{\text {dep }}(y)$ be given by

$$
\begin{aligned}
p_{\text {ind }}(y) & :=\sum_{x_{1}, x_{2}} p_{\text {ind }}\left(x_{1}, x_{2}\right) p\left(y \mid x_{1}, x_{2}\right) \\
p_{\text {dep }}(y) & :=\sum_{x_{1}, x_{2}} p_{\text {dep }}\left(x_{1}, x_{2}\right) p\left(y \mid x_{1}, x_{2}\right),
\end{aligned}
$$

respectively. For the distributions $p_{\text {ind }}(y)$ and $p_{\text {dep }}(y)$, we denote their KL divergence by

$$
D\left(p_{\text {dep }}(y) \| p_{\text {ind }}(y)\right):=\sum_{y} p_{\text {dep }}(y) \log \frac{p_{\text {dep }}(y)}{p_{\text {ind }}(y)} .
$$

Now let $\mathcal{C}^{*}$ be the class of all discrete MACs $\left(\mathcal{X}_{1} \times\right.$ $\left.\mathcal{X}_{2}, p\left(y \mid x_{1}, x_{2}\right), \mathcal{Y}\right)$ such that for a distribution $p_{\text {ind }}\left(x_{1}, x_{2}\right)=$ $p_{\text {ind }}\left(x_{1}\right) p_{\text {ind }}\left(x_{2}\right)$ that satisfies (2), there exists another distribution $p_{\text {dep }}\left(x_{1}, x_{2}\right)$ such that the support of $p_{\text {dep }}\left(x_{1}, x_{2}\right)$ is contained in the support of $p_{\text {ind }}\left(x_{1}\right) p_{\text {ind }}\left(x_{2}\right)$, and ${ }^{1}$

$$
I_{\text {dep }}\left(X_{1}, X_{2} ; Y\right)+D\left(p_{\text {dep }}(y) \| p_{\text {ind }}(y)\right)>I_{\text {ind }}\left(X_{1}, X_{2} ; Y\right) .
$$

Prior to discussing the significance of the class $\mathcal{C}^{*}$, we provide some examples. Consider a MAC with binary inputs $X_{1}, X_{2} \in\{0,1\}$. If $Y=X_{1} \oplus X_{2}$, where $\oplus$ is addition $\bmod 2$ and $Y \in\{0,1\}$, then the resulting MAC is not in $\mathcal{C}^{*}$. However, if $Y=X_{1}+X_{2}$, where + is integer addition and $Y \in\{0,1,2\}$, then the resulting MAC is in $\mathcal{C}^{*}$.

Theorem 1 below, which demonstrates the significance of $\mathcal{C}^{*}$, is a special case of [6, Theorem 3] for the two-user case ${ }^{2}$

Theorem 1. For any MAC in $\mathcal{C}^{*}$ and any $\left(\mathbf{C}_{\mathrm{in}}, \mathbf{v}\right) \in \mathbb{R}_{>0}^{2} \times$ $\mathbb{R}_{>0}^{2}$,

$$
\lim _{h \rightarrow 0^{+}} \frac{C_{\text {sum }}\left(\mathbf{C}_{\mathrm{in}}, h \mathbf{v}\right)-C_{\mathrm{sum}}\left(\mathbf{C}_{\mathrm{in}}, \mathbf{0}\right)}{h}=\infty .
$$

We next describe the maximal-error sum-capacity gain. While it is possible in the average-error scenario to achieve a sum-capacity that has an infinite slope, a stronger result is known in the maximal-error case. There exists a class of MACs for which the maximal-error sum-capacity exhibits a discontinuity in the capacities of the CF output links. This is stated formally in the next proposition, which is a special case of [5. Proposition 5]. The proposition relies on the existence of a discrete MAC with average-error sum-capacity larger than its maximal-error sum-capacity; that existence was first proven by Dueck [17]. We investigate further properties of Dueck's MAC in [5, Subsection VI-E].

Proposition 2. Consider a discrete MAC for which

$$
C_{\text {sum }}(\mathbf{0}, \mathbf{0})>C_{\text {sum, } \max }(\mathbf{0}, \mathbf{0}) .
$$

Fix $\mathbf{C}_{\mathrm{in}} \in \mathbb{R}_{>0}^{2}$. Then $C_{\mathrm{sum}, \max }\left(\mathbf{C}_{\mathrm{in}}, \mathbf{C}_{\text {out }}\right)$ is not continuous at $\mathbf{C}_{\text {out }}=\mathbf{0}$.

We next present the main results of this work.

\section{Our Results on Average- And Maximal-Error SUM-CAPACITIES}

As discussed in Section [1] we present our results for the large $\mathbf{C}_{\text {in }}$ (i.e., $\mathrm{CF}$ has full knowledge of the messages) and arbitrary $\mathbf{C}_{\text {in }}$ cases separately. We begin with the former case.

\footnotetext{
${ }^{1}$ As kindly demonstrated by one of the reviewers, the condition in 3 , can be simplified to $I_{\mathrm{dep}}\left(X_{1}, X_{2} ; Y\right)>I_{\text {ind }}\left(X_{1}, X_{2} ; Y\right)$. We provide their proof in the Appendix.

${ }^{2}$ Note that Theorem 1 does not lead to any conclusions regarding continuity; a function $f(x)$ with infinite derivative at $x=0$ can be continuous (e.g., $f(x)=\sqrt{x}$ ) or discontinuous (e.g., $f(x)=\lceil x\rceil$ ).
} 


\section{A. CF with Full Knowledge of the Messages}

Given a discrete $\operatorname{MAC}\left(\mathcal{X}_{1} \times \mathcal{X}_{2}, p\left(y \mid x_{1}, x_{2}\right), \mathcal{Y}\right)$, let the components of $\mathbf{C}_{\mathrm{in}}^{*}=\left(C_{\mathrm{in}}^{* 1}, C_{\mathrm{in}}^{* 2}\right)$ be sufficiently large so that any $\mathrm{CF}$ with input link capacities $C_{\mathrm{in}}^{* 1}$ and $C_{\mathrm{in}}^{* 2}$ has full knowledge of the encoders' messages. For example, we can choose $\mathbf{C}_{\mathrm{in}}^{*}$ such that

$$
\min \left\{C_{\mathrm{in}}^{* 1}, C_{\mathrm{in}}^{* 2}\right\}>\max _{p\left(x_{1}, x_{2}\right)} I\left(X_{1}, X_{2} ; Y\right) .
$$

Our first result, Theorem 3 , addresses the continuity of $C_{\text {sum }}\left(\mathbf{C}_{\text {in }}^{*}, \mathbf{C}_{\text {out }}\right)$ as a function of $\mathbf{C}_{\text {out }}$ over $\mathbb{R}_{\geq 0}^{2}$. We provide an outline of the proof in Section VI We also remark that while in this work we only consider discrete MACs, we expect similar ideas, when combined with techniques developed by Fong and Tan [18] that establish the strong converse for the Gaussian MAC, to extend to Gaussian MACs with a CF as well. The problem of continuity in the Gaussian case is left for future work.

Theorem 3. For any discrete MAC, the mapping

$$
\mathbf{C}_{\text {out }} \mapsto C_{\text {sum }}\left(\mathbf{C}_{\text {in }}^{*}, \mathbf{C}_{\text {out }}\right)
$$

defined on $\mathbb{R}_{>0}^{2}$ is continuous.

In words, Theorem 3 states that the average-error sumcapacity is continuous with respect to the CF output link capacities, a result that stands in stark contrast to the maximalerror case which we present next.

The second contribution of our work in the case where the $\mathrm{CF}$ has full knowledge of the messages is the discontinuity of the maximal-error sum-capacity when the CF can send only a constant number of bits back to the encoders. For this setting, we use Dueck's deterministic memoryless MAC [17], which we next define. Consider the deterministic MAC $\left(\mathcal{X}_{1} \times \mathcal{X}_{2}, p_{\text {Dueck }}\left(y \mid x_{1}, x_{2}\right), \mathcal{Y}\right)$ where $\mathcal{X}_{1}=\{a, b, A, B\}$, $\mathcal{X}_{2}=\{0,1\}$, and $\mathcal{Y}=\{a, b, c, A, B, C\} \times\{0,1\}$. Furthermore, $p_{\text {Dueck }}\left(y \mid x_{1}, x_{2}\right)$ equals one whenever $y=W\left(x_{1}, x_{2}\right)$ for the mapping $W: \mathcal{X}_{1} \times \mathcal{X}_{2} \rightarrow \mathcal{Y}$ and equals zero otherwise. The mapping $W$ is defined as

$$
W\left(x_{1}, x_{2}\right):= \begin{cases}(c, 0) & \text { if }\left(x_{1}, x_{2}\right) \in\{(a, 0),(b, 0)\} \\ (C, 1) & \text { if }\left(x_{1}, x_{2}\right) \in\{(A, 1),(B, 1)\} \\ \left(x_{1}, x_{2}\right) & \text { otherwise. }\end{cases}
$$

Theorem 4. For $k=5$, the discrete MAC $\left(\mathcal{X}_{1} \times\right.$ $\left.\mathcal{X}_{2}, p_{\text {Dueck }}\left(y \mid x_{1}, x_{2}\right), \mathcal{Y}\right)$ satisfies

$$
C_{\text {sum, } \max }\left(\mathbf{C}_{\text {in }}^{*}, k \text {-bit }\right)>C_{\text {sum, max }}\left(\mathbf{C}_{\text {in }}^{*}, \mathbf{0}\right) .
$$

We outline the proof of Theorem 4 in Section VII We provide detailed proofs of our claims in Section IX

\section{B. CF with Arbitrary Knowledge of the Messages}

In this subsection, we do not assume the CF has full knowledge of the messages and instead consider the general case. Our first result extends Theorem 1 by characterizing the growth rate of the MAC with $\mathrm{CF}$ average-error sum-capacity as a function of the CF output link capacities. We remark that a similar result to Theorem 5 below also holds for the Gaussian MAC [6, Prop. 9].
Theorem 5. Let $\left(\mathcal{X}_{1} \times \mathcal{X}_{2}, p\left(y \mid x_{1}, x_{2}\right), \mathcal{Y}\right)$ be a MAC in $\mathcal{C}^{*}$, and suppose $\left(\mathbf{C}_{\mathrm{in}}, \mathbf{v}\right) \in \mathbb{R}_{>0}^{2} \times \mathbb{R}_{>0}^{2}$. Then

$$
\liminf _{h \rightarrow 0^{+}} \frac{C_{\text {sum }}\left(\mathbf{C}_{\text {in }}, h \mathbf{v}\right)-C_{\text {sum }}\left(\mathbf{C}_{\text {in }}, \mathbf{0}\right)}{\sqrt{h}}>0 .
$$

In words, Theorem 5 states that for any MAC in $\mathcal{C}^{*}$, the average-error sum-capacity of that MAC with a $\mathrm{CF}$ grows at least as fast as the square root of the CF output link capacities. We prove this theorem in Subsection IX-A

We next describe the second result concerning averageerror sum-capacity. While our first result, Theorem 5 above, concerns the growth rate of $C_{\text {sum }}\left(\mathbf{C}_{\text {in }}, \mathbf{C}_{\text {out }}\right)$ near $\mathbf{C}_{\text {out }}=\mathbf{0}$ over $\mathbb{R}_{\geq 0}^{2}$, Theorem 6 proves the continuity of $C_{\text {sum }}\left(\mathbf{C}_{\text {in }}, \mathbf{C}_{\text {out }}\right)$ over several subsets of $\mathbb{R}_{\geq 0}^{2} \times \mathbb{R}_{\geq 0}^{2}$. We give the proof of this result in Section VIII.

Theorem 6. For any discrete MAC, consider the mapping

$$
\left(\tilde{\mathbf{C}}_{\text {in }}, \tilde{\mathbf{C}}_{\text {out }}\right) \mapsto C_{\text {sum }}\left(\tilde{\mathbf{C}}_{\text {in }}, \tilde{\mathbf{C}}_{\text {out }}\right),
$$

defined on $\mathbb{R}_{\geq 0}^{2} \times \mathbb{R}_{\geq 0}^{2}$. This mapping is continuous at $\left(\tilde{\mathbf{C}}_{\mathrm{in}}, \tilde{\mathbf{C}}_{\text {out }}\right)=\left(\mathbf{C}_{\mathrm{in}}, \mathbf{C}_{\text {out }}\right)$ if any of the following conditions hold:

a) $C_{\mathrm{in}}^{i}>0$ and $C_{\text {out }}^{i}>0$ for $i \in\{1,2\}$.

b) $C_{\text {out }}^{1}=0$ and $C_{\text {out }}^{2}=0$.

c) $C_{\text {in }}^{1} \geq C_{\text {in }}^{1 *}$ and $C_{\text {out }}^{2} \geq C_{\text {out }}^{2 *}$.

d) $C_{\mathrm{in}}^{1}=0$ or $C_{\mathrm{in}}^{2}=0$.

Note that Theorem 6 leaves open the continuity of the average-error sum-capacity in one case. If $C_{\mathrm{in}}^{1}$ and $C_{\mathrm{in}}^{2}$ are positive but not sufficiently large and $C_{\text {out }}^{1}>0$, then we have not established the continuity of the sum-capacity at $C_{\text {out }}^{2}=0^{+}$. (Clearly, the symmetric case where $C_{\text {out }}^{2}>0$ and $C_{\text {out }}^{1} \rightarrow 0^{+}$remains open as well.) This last scenario remains a subject for future work. The corollary below, however, demonstrates that to prove the continuity of the average-error sum-capacity $C_{\text {sum }}$ at these remaining points, we may fix $\mathbf{C}_{\text {in }}$ and one of $C_{\text {out }}^{1}$ or $C_{\text {out }}^{2}$, meaning that we only need to show the continuity of $C_{\text {sum }}$ in a single variable. We prove this corollary in Subsection IX-B.

Corollary 7. For any discrete $M A C$, to prove that the mapping

$$
\left(\mathbf{C}_{\text {in }}, \mathbf{C}_{\text {out }}\right) \mapsto C_{\text {sum }}\left(\mathbf{C}_{\text {in }}, \mathbf{C}_{\text {out }}\right),
$$

defined on $\mathbb{R}_{>0}^{2} \times \mathbb{R}_{>0}^{2}$ is continuous, it suffices to show that for all $\left(\mathbf{C}_{\text {in }}, \mathbf{C}_{\text {out }}\right) \in \mathbb{R}_{>0}^{2} \times \mathbb{R}_{>0}^{2}$, we have

$$
C_{\text {sum }}\left(\mathbf{C}_{\text {in }},\left(\tilde{C}_{\text {out }}^{1}, C_{\text {out }}^{2}\right)\right) \rightarrow C_{\text {sum }}\left(\mathbf{C}_{\text {in }},\left(0, C_{\text {out }}^{2}\right)\right)
$$

as $\tilde{C}_{\text {out }}^{1} \rightarrow 0^{+}$and

$$
C_{\text {sum }}\left(\mathbf{C}_{\text {in }},\left(C_{\text {out }}^{1}, \tilde{C}_{\text {out }}^{2}\right)\right) \rightarrow C_{\text {sum }}\left(\mathbf{C}_{\text {in }},\left(C_{\text {out }}^{1}, 0\right)\right)
$$

as $\tilde{C}_{\text {out }}^{2} \rightarrow 0^{+}$.

Finally, we describe our result for the maximal-error sumcapacity for an arbitrary CF. Recall that Proposition 2 gives a sufficient condition under which $C_{\text {sum, max }}\left(\mathbf{C}_{\text {in }}, \mathbf{C}_{\text {out }}\right)$ is not continuous at $\mathbf{C}_{\text {out }}=\mathbf{0}$ for a fixed $\mathbf{C}_{\mathrm{in}} \in \mathbb{R}_{>0}^{2}$. In Subsection IX-C we show that the sufficient condition is also necessary. This is stated in the next theorem. 
Theorem 8. Fix a discrete $M A C$ and $\mathbf{C}_{\text {in }} \in \mathbb{R}_{>0}^{2}$. Then $C_{\text {sum, max }}\left(\mathbf{C}_{\text {in }}, \mathbf{C}_{\text {out }}\right)$ is not continuous at $\mathbf{C}_{\text {out }}=\mathbf{0}$ if and only if

$$
C_{\text {sum }}(\mathbf{0}, \mathbf{0})>C_{\text {sum, } \max }(\mathbf{0}, \mathbf{0}) .
$$

\section{Average-ERror Sum-CAPACITy Continuity: CF WITH FULL KNOWLEDGE}

In this section, we give an outline for the proof of Theorem 3 We start our study of the continuity of $C_{\text {sum }}\left(\mathbf{C}_{\mathrm{in}}^{*}, \mathbf{C}_{\text {out }}\right)$ by presenting lower and upper bounds in terms of an auxiliary function $\sigma(\delta)$ defined for $\delta \geq 0$ (Lemma 10). This function is similar to a tool used by Dueck [19] but is slightly different in its reliance on a time-sharing random variable denoted by $U$. The random variable $U$ plays two roles. First it ensures that $\sigma$ is concave, which immediately proves the continuity of $\sigma$ over $\mathbb{R}_{>0}$. Second, together with a lemma from [19] (Lemma 13 below), it helps us find a single-letter upper bound for $\sigma$ (Corollary 14). We then use that upper bound to prove continuity at $\delta=0$. We remark that Dueck's technique [19] is used by a number of other authors as well in related contexts, including Saeedi Bidokhti and Kramer [20] and Kosut and Kliewer [13, Proposition 15]. We first use this technique for the continuity problem in [14. Appendix C].

The following definitions are useful for the description of our lower and upper bounds for $C_{\text {sum }}\left(\mathbf{C}_{\text {in }}^{*}, \mathbf{C}_{\text {out }}\right)$. For every finite alphabet $\mathcal{U}$ and all $\delta \geq 0$, define the set of probability mass functions $\mathcal{P}_{\mathcal{U}}^{(n)}(\delta)$ on $\mathcal{U} \times \mathcal{X}_{1}^{n} \times \mathcal{X}_{2}^{n}$ as

$$
\mathcal{P}_{\mathcal{U}}^{(n)}(\delta):=\left\{p\left(u, x_{1}^{n}, x_{2}^{n}\right) \mid I\left(X_{1}^{n} ; X_{2}^{n} \mid U\right) \leq n \delta\right\} .
$$

Intuitively, $\mathcal{P}_{\mathcal{U}}^{(n)}(\delta)$ captures a family of "mildly dependent" input distributions for our MAC; this mild dependence is parametrized by a bound $\delta$ on the per-symbol mutual information. In the discussion that follows, we relate $\delta$ to the amount of information that the $\mathrm{CF}$ shares with the encoders. For every positive integer $n$, let $\sigma_{n}: \mathbb{R}_{\geq 0} \rightarrow \mathbb{R}_{\geq 0}$ denote the function 3

$$
\sigma_{n}(\delta):=\sup _{\mathcal{U}} \max _{p \in \mathcal{P}_{\mathcal{U}}^{(n)}(\delta)} \frac{1}{n} I\left(X_{1}^{n}, X_{2}^{n} ; Y^{n} \mid U\right),
$$

where the supremum is over all finite sets $\mathcal{U}$. Thus $\sigma_{n}(\delta)$ captures something like the maximal sum-rate achievable under the mild dependence described above. As we see in Lemma 12, conditioning on the random variable $U$ in 8 ensures that $\sigma_{n}$ is concave.

For every $\delta \geq 0,\left(\sigma_{n}(\delta)\right)_{n=1}^{\infty}$ satisfies a superadditivity property which appears in Lemma 9, below. Intuitively, this property says that the sum-rate of the best code of blocklength $m+n$ is bounded from below by the sum-rate of the concatenation of the best codes of blocklengths $m$ and $n$. We prove this Lemma in Subsection IX-D

Lemma 9. For all $m, n \geq 1$, all $\delta \geq 0$, and $\sigma_{n}(\delta)$ defined as in (8), we have

$$
(m+n) \sigma_{m+n}(\delta) \geq m \sigma_{m}(\delta)+n \sigma_{n}(\delta) .
$$

\footnotetext{
${ }^{3}$ For $n=1$, this function also appears in the study of the MAC with negligible feedback [7].
}

Given Lemma 9, [21, Appendix 4A, Lemma 2] now implies that the sequence of mappings $\left(\sigma_{n}\right)_{n=1}^{\infty}$ converges pointwise to some mapping $\sigma: \mathbb{R}_{\geq 0} \rightarrow \mathbb{R}_{\geq 0}$, and

$$
\sigma(\delta):=\lim _{n \rightarrow \infty} \sigma_{n}(\delta)=\sup _{n} \sigma_{n}(\delta) .
$$

We next present our lower and upper bounds for $C_{\text {sum }}\left(\mathbf{C}_{\text {in }}^{*}, \mathbf{C}_{\text {out }}\right)$ in terms of $\sigma$. The lower bound follows directly from [6. Corollary 8]. We prove the upper bound in Subsection IX-E

Lemma 10. For any discrete $M A C$ and any $\mathbf{C}_{\text {out }} \in \mathbb{R}_{\geq 0}^{2}$, we have

$$
\begin{aligned}
\sigma\left(C_{\text {out }}^{1}+C_{\text {out }}^{2}\right)-\min \left\{C_{\text {out }}^{1}, C_{\text {out }}^{2}\right\} & \leq C_{\text {sum }}\left(\mathbf{C}_{\text {in }}^{*}, \mathbf{C}_{\text {out }}\right) \\
& \leq \sigma\left(C_{\text {out }}^{1}+C_{\text {out }}^{2}\right) .
\end{aligned}
$$

From the remark following Theorem 6, we only need to prove that $C_{\text {sum }}\left(\mathbf{C}_{\text {in }}^{*}, \mathbf{C}_{\text {out }}\right)$ is continuous on the boundary of $\mathbb{R}_{>0}^{2}$. On the boundary of $\mathbb{R}_{>0}^{2}$, however, $\min \left\{C_{\text {out }}^{1}, C_{\text {out }}^{2}\right\}=$ 0 . Thus it suffices to show that $\sigma$ is continuous on $\mathbb{R}_{\geq 0}$, which is proven in the next lemma.

Lemma 11. For any finite alphabet MAC, the function $\sigma$, defined by (9), is continuous on $\mathbb{R}_{\geq 0}$.

To prove Lemma 11, we first consider the continuity of $\sigma$ on $\mathbb{R}_{>0}$ and then focus on the point $\delta=0^{+}$. Note that $\sigma$ is the pointwise limit of the sequence of functions $\left(\sigma_{n}\right)_{n=1}^{\infty}$. Lemma 12 uses a time-sharing argument as in [22] to show that each $\sigma_{n}$ is concave. (See Subsection IX-F for the proof.) Therefore, $\sigma$ is concave as well, and since $\mathbb{R}_{>0}$ is open, $\sigma$ is continuous on $\mathbb{R}_{>0}$.

\section{Lemma 12. For all $n \geq 1, \sigma_{n}$ is concave on $\mathbb{R}_{\geq 0}$.}

To prove the continuity of $\sigma(\delta)$ at $\delta=0$, we find an upper bound for $\sigma$ in terms of $\sigma_{1}$. For some finite set $\mathcal{U}$ and $\delta>0$, consider a distribution $p\left(u, x_{1}^{n}, x_{2}^{n}\right) \in \mathcal{P}_{\mathcal{U}}^{(n)}(\delta)$. By the definition of $\mathcal{P}_{\mathcal{U}}^{(n)}(\delta)$,

$$
I\left(X_{1}^{n} ; X_{2}^{n} \mid U\right) \leq n \delta .
$$

Finding a bound for $\sigma$ in terms of $\sigma_{1}$ requires a single-letter version of [10]. In [19, Subsection II.D], Dueck presents the necessary result. We present Dueck's result in the next lemma and provide the proof in Subsection IX-G for completeness.

Lemma 13 (Dueck's Lemma [19]). Fix positive real numbers $\epsilon$ and $\delta$, positive integer $n$, and finite alphabet $\mathcal{U}$. If $p \in$ $\mathcal{P}_{\mathcal{U}}^{(n)}(\delta)$, then there exists a set $T \subseteq[n]$ satisfying $|T| \leq n \delta / \epsilon$ such that

$$
\forall t \notin T: I\left(X_{1 t} ; X_{2 t} \mid U, X_{1}^{T}, X_{2}^{T}\right) \leq \epsilon,
$$

where for $i \in\{1,2\}, X_{i}^{T}:=\left(X_{i t}\right)_{t \in T}$.

Corollary 14 uses Lemma 13 to find an upper bound for $\sigma$ in terms of $\sigma_{1}$. The proof of this corollary (see Subsection [X-H) combines ideas from [19] with results derived here.

Corollary 14. For all $\epsilon, \delta>0$, we have

$$
\sigma(\delta) \leq \frac{\delta}{\epsilon} \log \left|\mathcal{X}_{1}\right|\left|\mathcal{X}_{2}\right|+\sigma_{1}(\epsilon)
$$


By Corollary 14 we have

$$
\sigma(0) \leq \lim _{\delta \rightarrow 0^{+}} \sigma(\delta) \leq \sigma_{1}(\epsilon) .
$$

If we calculate the limit $\epsilon \rightarrow 0^{+}$, we get

$$
\sigma(0) \leq \lim _{\delta \rightarrow 0^{+}} \sigma(\delta) \leq \lim _{\epsilon \rightarrow 0^{+}} \sigma_{1}(\epsilon) .
$$

Since $\sigma(0)=\sigma_{1}(0) 4^{4}$ it suffices to show that $\sigma_{1}(\delta)$ is continuous at $\delta=0^{+}$. Recall that $\sigma_{1}$ is defined as

$$
\sigma_{1}(\delta):=\sup _{\mathcal{U}} \max _{p \in \mathcal{P}_{\mathcal{U}}^{(1)}(\delta)} I\left(X_{1}, X_{2} ; Y \mid U\right) .
$$

Since the supremum in (11) is over all finite sets $\mathcal{U}$, it is difficult to find an upper bound for $\sigma_{1}(\delta)$ near $\delta=0^{+}$directly. Instead we first show, in Subsection [X-1, that it is possible to assume that $\mathcal{U}$ has at most two elements.

Lemma 15 (Cardinality of $\mathcal{U}$ ). For any positive integer $n$, in the definition of $\sigma_{n}(\delta)$, it suffices to let $\mathcal{U}$ be any two-element set; that is, for $\mathcal{U}:=\{1,2\}$, we can write

$$
\sigma_{n}(\delta)=\max _{p \in \mathcal{P}_{\mathcal{U}}^{(n)}(\delta)} \frac{1}{n} I\left(X_{1}^{n}, X_{2}^{n} ; Y^{n} \mid U\right) .
$$

In Subsection [X-J, we prove the continuity of $\sigma_{1}$ at $\delta=$ $0^{+}$from Lemma 15 using standard tools, such as Pinsker's inequality [3. Lemma 17.3.3] and the $L_{1}$ lower bound of KL divergence [3, Lemma 11.6.1]. The continuity of $\sigma_{1}$ on $\mathbb{R}_{>0}$ follows from the concavity of $\sigma_{1}$ on $\mathbb{R}_{\geq 0}$.

Lemma 16 (Continuity of $\sigma_{1}$ ). The function $\sigma_{1}$ is continuous on $\mathbb{R}_{\geq 0}$.

\section{MAXIMAL-ERROR SUM-CAPACITY DiscontinUity: CONSTANT Number OF COOPERATION BITS}

In this section we prove Theorem 4 We use Dueck's [17] deterministic memoryless MAC $\left(\mathcal{X}_{1} \times \mathcal{X}_{2}, p_{\text {Dueck }}\left(y \mid x_{1}, x_{2}\right), \mathcal{Y}\right)$ where $\mathcal{X}_{1}, \mathcal{X}_{2}, \mathcal{Y}, p_{\text {Dueck }}$, and $W$ are as defined in Section $\mathrm{V}$. For positive integer $n$, we define the mapping $W^{n}: \mathcal{X}_{1}^{n} \times \mathcal{X}_{2}^{n} \rightarrow \mathcal{Y}^{n}$ as $\left(y_{1}^{n}, y_{2}^{n}\right)=W^{n}\left(x_{1}^{n}, x_{2}^{n}\right)$ if and only if for all $1 \leq i \leq n$,

$$
\left(y_{1 i}, y_{2 i}\right)=W\left(x_{1 i}, x_{2 i}\right) \text {. }
$$

Set $\mathbf{C}_{\text {in }}^{*}=\left(\log \left|\mathcal{X}_{1}\right|, \log \left|\mathcal{X}_{2}\right|\right)=(2,1)$. This suffices to enable the $\mathrm{CF}$ to have access to both source messages $w_{1}$ and $w_{2}$. We use the following theorem from [17].

Theorem 17 (Outer Bound on the Maximal-Error Sum-Capacity [17]). For the MAC $\left(\mathcal{X}_{1} \times\right.$ $\left.\mathcal{X}_{2}, p_{\text {Dueck }}\left(y \mid x_{1}, x_{2}\right), \mathcal{Y}\right)$, we have

$$
C_{\text {sum,max }}(\mathbf{0}, \mathbf{0}) \leq \max _{0 \leq p \leq 1 / 2}[H(1 / 3)+2 / 3-p+H(p)] .
$$

Optimizing over $p$, and noting that $C_{\text {sum, } \max }(\mathbf{0}, \mathbf{0})=$ $C_{\text {sum,max }}\left(\mathbf{C}_{\text {in }}^{*}, \mathbf{0}\right)$, Theorem 17 directly implies the following corollary.

\footnotetext{
${ }^{4}$ This follows from the converse proof of the MAC capacity region in the absence of cooperation [3 Theorem 15.3.1].
}

Corollary 18. For the MAC $\left(\mathcal{X}_{1} \times \mathcal{X}_{2}, p_{\text {Dueck }}\left(y \mid x_{1}, x_{2}\right), \mathcal{Y}\right)$ and $p^{*}=1 / 3$, we have

$$
C_{\text {sum }, \max }\left(\mathbf{C}_{\text {in }}^{*}, \mathbf{0}\right) \leq H\left(p^{*}\right)+H(1 / 3)+2 / 3-p^{*} \leq 2.1699 .
$$

To conclude the proof of Theorem 4, we now show that for $k=5, C_{\text {sum, max }}\left(\mathbf{C}_{\text {in }}^{*}, k\right.$-bit $)>C_{\text {sum, max }}\left(\mathbf{C}_{\text {in }}^{*}, \mathbf{0}\right)$. Specifically, for any $\epsilon>0, \delta>0, k=\lceil\log \lceil 3 / \delta\rceil\rceil+1$, and $n$ sufficiently large, we define a $\left(2^{n R_{1}}, 2^{n R_{2}}, n\right)$-code for our MAC with a $\left(\mathbf{C}_{\text {in }}^{*}, k\right.$-bit $)$-CF with maximal error zero in which $R_{1}=(1.5-\delta)(1-\epsilon)$ and $R_{2}=1-\epsilon$. Corollary 18 then implies that for $\delta=1 / 4, k=5$, and $\epsilon>0$ sufficiently small, we have $C_{\text {sum, } \max }\left(\mathbf{C}_{\mathrm{in}}^{*}, k\right.$-bit $\left.)\right)>C_{\text {sum, } \max }\left(\mathbf{C}_{\mathrm{in}}^{*}, \mathbf{0}\right)$. We specify our code by presenting the functions $\left(\varphi_{1}, \varphi_{2}, \psi_{1}, \psi_{2}, f_{1}, f_{2}, g\right)$.

The functions $\varphi_{1}$ and $\varphi_{2}$ are the identity functions. Function $\psi_{1}$ is a constant function which always returns $0^{k}$. That is, for our construction, the $\mathrm{CF}$ only needs to send the cooperation information to encoder 2. Thus the function $f_{1}$ does not depend on $\psi_{1}$ and maps message $w_{1}$ to $x_{1}^{n}$ deterministically according to a codebook $\left\{x_{1}^{n}\left(w_{1}\right)\right\}_{w_{1} \in\left[2^{\left.n R_{1}\right]}\right.}$ to be specified later. Before we define the codebook $\left\{x_{1}^{n}\left(w_{1}\right)\right\}_{w_{1} \in\left[2^{\left.n R_{1}\right]}\right.}$, and functions $\psi_{2}, f_{2}$, and $g$, we introduce some notation and definitions.

Recall that $\mathcal{Y}=\{a, b, c, A, B, C\} \times\{0,1\}$. We can thus write $\mathcal{Y}=\mathcal{Y}_{1} \times \mathcal{Y}_{2}$, where $\mathcal{Y}_{1}=\{A, B, C, a, b, c\}$ and $\mathcal{Y}_{2}=\{0,1\}$. Accordingly, let $y_{1}^{n}=\left(y_{1,1}, \ldots, y_{1, n}\right) \in \mathcal{Y}_{1}^{n}$ and $y_{2}^{n}=\left(y_{2,1}, \ldots, y_{2, n}\right) \in \mathcal{Y}_{2}^{n}$. Given transmitted codewords $x_{1}^{n}$ and $x_{2}^{n}$, recall that $\left(y_{1}^{n}, y_{2}^{n}\right)=W^{n}\left(x_{1}^{n}, x_{2}^{n}\right)$, where $W^{n}$ is the blocklength- $n$ extension of $W$ defined above. We use notation $y_{1}^{n}=W_{1}^{n}\left(x_{1}^{n}, x_{2}^{n}\right)$ and $y_{2}^{n}=W_{2}^{n}\left(x_{1}^{n}, x_{2}^{n}\right)$ to describe $W^{n}$. Formally, we use $W\left(x_{1}, x_{2}\right)=\left(W_{1}\left(x_{1}, x_{2}\right), W_{2}\left(x_{1}, x_{2}\right)\right)$. Note that $y_{2}^{n}=W_{2}^{n}\left(x_{1}^{n}, x_{2}^{n}\right)=x_{2}^{n}$ for all $x_{1}^{n} \in \mathcal{X}_{1}^{n}$.

Our communication scheme is divided into two phases, the first of blocklength $n_{1}=(1-\epsilon) n$, and the second of blocklength $n_{2}=\epsilon n$, with $n=n_{1}+n_{2}$. Roughly speaking, after the first phase the decoder is able to listdecode the message $\left(w_{1}, w_{2}\right)$, and after the second it is able to determine the correct message from its list. We thus refine our notation, and define for $i=1,2: x_{i}^{n_{1}}=\left(x_{i, 1}, \ldots, x_{i, n_{1}}\right)$, $x_{i}^{n_{2}}=\left(x_{i, n_{1}+1}, \ldots, x_{i, n}\right), y_{i}^{n_{1}}=\left(y_{i, 1}, \ldots, y_{i, n_{1}}\right)$, and $y_{i}^{n_{2}}=$ $\left(y_{i, n_{1}+1}, \ldots, y_{i, n}\right)$. Accordingly, we represent the two phases in the encoding functions $f_{1}$ and $f_{2}$ as $f_{1,1}, f_{1,2}$ and $f_{2,1}, f_{2,2}$. Namely, for messages $w_{1}$ and $w_{2}$ we have $x_{1}^{n_{1}}=f_{1,1}\left(w_{1}\right)$, $x_{1}^{n_{2}}=f_{1,2}\left(w_{1}\right), x_{2}^{n_{1}}=f_{2,1}\left(w_{2}, \psi_{2}\left(w_{1}, w_{2}\right)\right)$, and $x_{2}^{n_{2}}=$ $f_{2,2}\left(w_{2}, \psi_{2}\left(w_{1}, w_{2}\right)\right)$. We start by discussing the first phase of communication.

Consider a vector $y^{n_{1}}=\left(y_{1}^{n_{1}}, y_{2}^{n_{1}}\right)$. Let $\mathcal{E}\left(y_{1}^{n_{1}}\right)=\mid\{i \mid$ $\left.y_{1, i}^{n} \in\{c, C\}\right\} \mid$ be the number of symbols in $y_{1}^{n_{1}}$ that equal $c$ or $C$. Note that both $c$ and $C$ are the result of an erasure, since the channel maps both $a$ and $b$, or both $A$ and $B$, to the same output ${ }^{5}$ Let $\left(W^{-1}\right)^{n_{1}}\left(y_{1}^{n_{1}}, y_{2}^{n_{1}}\right)$ be the set of inputs $\left(x_{1}^{n_{1}}, x_{2}^{n_{1}}\right) \in \mathcal{X}_{1}^{n_{1}} \times \mathcal{X}_{2}^{n_{1}}$ for which $W^{n_{1}}\left(x_{1}^{n_{1}}, x_{2}^{n_{1}}\right)=$ $\left(y_{1}^{n_{1}}, y_{2}^{n_{1}}\right)$; then from the definition of our channel it holds that $\left|\left(W^{-1}\right)^{n_{1}}\left(y_{1}^{n_{1}}, y_{2}^{n_{1}}\right)\right|=2^{\mathcal{E}\left(y_{1}^{n_{1}}\right)}$ since each erasure symbol must have resulted from one of two possible input symbols.

\footnotetext{
${ }^{5}$ Since output $Y_{1}=c$ occurs only with output $Y_{2}=0$ and output $Y_{1}=C$ occurs only with output $Y_{2}=1$, we could, alternatively, denote both by the erasure symbol " $E$ " without losing any information.
} 
We say that the pair $\left(y_{1}^{n_{1}}, y_{2}^{n_{1}}\right)$ is good if $\mathcal{E}\left(y_{1}^{n_{1}}\right) \leq n_{1} / 2$ or equivalently, $\left|\left(W^{-1}\right)^{n_{1}}\left(y_{1}^{n_{1}}, y_{2}^{n_{1}}\right)\right| \leq 2^{n_{1} / 2}$; thus the first phase of a channel output is good if at most half of the input symbols are erased. In our encoding scheme, we would like to guarantee that $\left(y_{1}^{n_{1}}, y_{2}^{n_{1}}\right)$ is always good. This is accomplished using the cooperation bits of $\psi_{2}$, or more specifically, using the first bit $\psi_{2,1}$ of $\psi_{2}$. Note that since we are interested in designing a code with $R_{2}=1-\epsilon$, we may represent $w_{2}$ as a binary vector of length $n(1-\epsilon)$. With this representation in mind, we specify $\psi_{2,1}$. The remaining bits of $\psi_{2}$ are defined as

$$
\psi_{2,1}\left(w_{1}, w_{2}\right)= \begin{cases}0 & \text { if } W^{n_{1}}\left(f_{1,1}\left(w_{1}\right), w_{2}\right) \text { is good, } \\ 1 & \text { otherwise. }\end{cases}
$$

Note that our MAC and $f_{1,1}$ are deterministic, and thus the CF can compute $W^{n_{1}}\left(f_{1,1}\left(w_{1}\right), w_{2}\right)$.

We are now ready to define the first phase of encoding, namely $f_{1,1}$ and $f_{2,1}$. We start with $f_{2,1}$ which equals either $w_{2}$ or its element-wise complement $\bar{w}_{2}$.

$$
f_{2,1}\left(w_{2}, \psi_{2,1}\left(w_{1}, w_{2}\right)\right)= \begin{cases}w_{2} & \text { if } \psi_{2,1}\left(w_{1}, w_{2}\right)=0, \\ \bar{w}_{2} & \text { otherwise. }\end{cases}
$$

Since $\psi_{2,1}\left(w_{1}, w_{2}\right)$ is 0 when $W^{n_{1}}\left(f_{1,1}\left(w_{1}\right), w_{2}\right)$ is good and 1 otherwise, $f_{2: 1}$ flips the message $w_{2}$ if and only if the unflipped channel input yields a bad channel output. The following claim, which we prove in Subsection IX-K, now guarantees that $y^{n_{1}}=\left(y_{1}^{n_{1}}, y_{2}^{n_{1}}\right)$ is good.

Claim 19. For any $w_{1}$ and $w_{2}$, and any deterministic mapping $f_{1,1}$, if $W^{n_{1}}\left(f_{1,1}\left(w_{1}\right), w_{2}\right)$ is bad then $W^{n_{1}}\left(f_{1: 1}\left(w_{1}\right), \bar{w}_{2}\right)$ is good.

We now define $f_{1,1}$, i.e., the codebook $\left\{x_{1}^{n_{1}}\left(w_{1}\right)\right\}_{w_{1} \in\left[2^{n R_{1}}\right]}$. Consider first choosing the codebook uniformly at random from all subsets of size $2^{n R_{1}}$ of $\mathcal{X}_{1}^{n_{1}}$. Namely, let the function $f_{1,1}$ be distributed uniformly over all injective functions $\left[2^{n R_{1}}\right] \rightarrow \mathcal{X}_{1}^{n_{1}}$. We show that with high probability over $f_{1,1}$, for any good received word $\left(y_{1}^{n_{1}}, y_{2}^{n_{1}}\right)$, there are at most $2\lceil 3 / \delta\rceil$ codeword pairs $\left(x_{1}^{n_{1}}, x_{2}^{n_{1}}\right)$ that satisfy $W^{n_{1}}\left(x_{1}^{n_{1}}, x_{2}^{n_{1}}\right)=\left(y_{1}^{n_{1}}, y_{2}^{n_{1}}\right)$, where $\delta$ is defined in our choice of $R_{1}$ and is independent of the blocklength $n_{1}$.

Claim 20. For any sufficiently large $n_{1}$, with probability at least $1-2^{-0.4 n_{1}}$ over $f_{1,1}$, for any good pair $\left(y_{1}^{n_{1}}, y_{2}^{n_{1}}\right)$, there are at most $\lceil 3 / \delta\rceil$ messages $w_{1}$ such that $W^{n_{1}}\left(f_{1,1}\left(w_{1}\right), y_{2}^{n_{1}}\right)=\left(y_{1}^{n_{1}}, y_{2}^{n_{1}}\right)$.

We prove Claim 20 in Subsection IX-L.

Consider any function $f_{1,1}$ that satisfies the conditions of Claim 20 Function $f_{1,1}$ defines the codebook $\left\{x_{1}^{n_{1}}\left(w_{1}\right)\right\}_{w_{1} \in\left[2^{n R_{1}}\right]}$ by setting $x_{1}^{n_{1}}\left(w_{1}\right)=f_{1,1}\left(w_{1}\right)$. By Claim 19 and our definition of $\psi_{2,1}\left(w_{1}, w_{2}\right)$, for any message pair $\left(w_{1}, w_{2}\right), \quad W^{n_{1}}\left(f_{1,1}\left(w_{1}\right), f_{2,1}\left(w_{2}, \psi_{2,1}\left(w_{1}, w_{2}\right)\right)\right)=$ $\left(y_{1}^{n_{1}}, y_{2}^{n_{1}}\right)$ is always good. Consider a preliminary decoding function $g_{\text {list }}$ that, given $y^{n_{1}}=\left(y_{1}^{n_{1}}, y_{2}^{n_{1}}\right)$, returns all possible $w_{1}$ such that $W^{n_{1}}\left(f_{1,1}\left(w_{1}\right), y_{2}^{n_{1}}\right)=\left(y_{1}^{n_{1}}, y_{2}^{n_{1}}\right)$. By Claim 20 . the decoder $g_{\text {list }}$ is a list-decoder with list size $\lceil 3 / \delta\rceil$. Here, a list-decoder is a decoding function that returns a list of potential messages (of a limited size) which, under our deterministic channel model and code design, is guaranteed to include the original source message $w_{1}$.
We thus conclude that for $R_{1}=(1.5-\delta)(1-\epsilon)$ and $R_{2}=1-\epsilon$, after the first phase of communication, by Claim 20 the decoder can recover a list $L$ of size $\ell=\lceil 3 / \delta\rceil$ of potential $w_{1}$ of encoder 1 , and a list of size 2 of potential messages $w_{2}$ of encoder 2. The latter follows from our channel model that guarantees that either $w_{2}$ or $\bar{w}_{2}$ equal $y_{2}^{n_{1}}$. Moreover, we note, due to the deterministic nature of our channel, that the CF can calculate the list $L$ and the location (in lexicographic order) of the original message $w_{1}$ in the list. As the list size for $w_{1}$ is $\ell,\lceil\log \ell\rceil=\lceil\log \lceil 3 / \delta\rceil\rceil$ bits suffice to specify the original message $w_{1}$ from the received pair $\left(y_{1}^{n_{1}}, y_{2}^{n_{1}}\right)$. We therefore define the remaining bits of $\psi_{2}\left(w_{1}, w_{2}\right)$, denoted by $\psi_{2,2}\left(w_{1}, w_{2}\right)$, to be the binary representation of the location of the original message $w_{1}$ in the list $L$. Note that, given $\left(y_{1}^{n_{1}}, y_{2}^{n_{1}}\right)$, the knowledge of $\psi_{2}\left(w_{1}, w_{2}\right)=\left(\psi_{2,1}\left(w_{1}, w_{2}\right), \psi_{2,2}\left(w_{1}, w_{2}\right)\right)$ allows the decoder to recover the original message pair $\left(w_{1}, w_{2}\right)$. The message $w_{1}$ is recovered from list $L$ via $\psi_{2,2}\left(w_{1}, w_{2}\right)$ and the message $w_{2}$ is recovered from $y_{2}^{n_{1}}$ via $\psi_{2,1}\left(w_{1}, w_{2}\right)$. We now show how encoders 1 and 2 can send $\psi_{2}\left(w_{1}, w_{2}\right)$ to the decoder in the second phase of our communication scheme.

To send $\psi_{2}\left(w_{1}, w_{2}\right)$ to the decoder during the second phase of communication (of blocklegth $n_{2}=\epsilon n$ ) we use the fact that the rate pair $(0,1)$ is in the zero error capacity region of our MAC. This follows by noticing that $W_{2}\left(x_{1}, x_{2}\right)=x_{2}$. Thus, we set $f_{1,2}\left(w_{1}\right)$ to be constant and $f_{2,2}\left(w_{2}, \psi_{2}\left(w_{1}, w_{2}\right)\right)$ to equal $\psi_{2}\left(w_{1}, w_{2}\right)$ padded on the left by zeros (here, we use the fact that for sufficiently large $n$ it holds that $n_{2}=\epsilon n \geq$ $\lceil\log \lceil 3 / \delta\rceil\rceil+1)$. The decoder can now recover $\psi_{2}\left(w_{1}, w_{2}\right)$ and in turn the original message pair $\left(w_{1}, w_{2}\right)$ from its list. This concludes the proof of Theorem 4

\section{AVERAGE-ERror SUM-CAPACITY CONTINUITY: GENERAL CF}

In this section, we study the continuity of $C_{\text {sum }}\left(\mathbf{C}_{\text {in }}, \mathbf{C}_{\text {out }}\right)$ with the aim of proving Theorem 6

Case (a): $C_{\text {in }}^{i}>0$ and $C_{\text {out }}^{i}>0$ for $i \in\{1,2\}$. Using a time-sharing argument, it is possible to show that $C_{\text {sum }}$ is concave on $\mathbb{R}_{\geq 0}^{2} \times \mathbb{R}_{\geq 0}^{2}$ and thus continuous on its interior [23 Lemma 4]. Hence $C_{\text {sum }}$ is continuous at any point $\left(\mathbf{C}_{\text {in }}, \mathbf{C}_{\text {out }}\right)$ where $C_{\mathrm{in}}^{1}, C_{\mathrm{in}}^{2}, C_{\text {out }}^{1}$, and $C_{\text {out }}^{2}$ are all positive.

For the remaining cases, we need an auxiliary result which we next discuss.

Fix $\left(\mathbf{C}_{\text {in }}, \mathbf{C}_{\text {out }}\right)$. For arbitrary $\left(\tilde{\mathbf{C}}_{\text {in }}, \tilde{\mathbf{C}}_{\text {out }}\right)$, the triangle inequality implies

$$
\begin{aligned}
& \left|C_{\text {sum }}\left(\tilde{\mathbf{C}}_{\text {in }}, \tilde{\mathbf{C}}_{\text {out }}\right)-C_{\text {sum }}\left(\mathbf{C}_{\text {in }}, \mathbf{C}_{\text {out }}\right)\right| \\
& \leq|| C_{\text {sum }}\left(\tilde{\mathbf{C}}_{\text {in }}, \tilde{\mathbf{C}}_{\text {out }}\right)-C_{\text {sum }}\left(\mathbf{C}_{\text {in }}, \tilde{\mathbf{C}}_{\text {out }}\right) \mid \\
& \quad+\left|C_{\text {sum }}\left(\mathbf{C}_{\text {in }}, \tilde{\mathbf{C}}_{\text {out }}\right)-C_{\text {sum }}\left(\mathbf{C}_{\text {in }}, \mathbf{C}_{\text {out }}\right)\right| .
\end{aligned}
$$

We study this bound in the limit $\left(\tilde{\mathbf{C}}_{\text {in }}, \tilde{\mathbf{C}}_{\text {out }}\right) \rightarrow\left(\mathbf{C}_{\text {in }}, \mathbf{C}_{\text {out }}\right)$. To begin, we consider the first term in (13).

Lemma 21 (Continuity of Sum-Capacity in $\mathbf{C}_{\mathrm{in}}$ ). There exists a function

$$
\Delta: \mathbb{R}_{\geq 0}^{2} \times \mathbb{R}_{\geq 0}^{2} \rightarrow \mathbb{R}_{\geq 0}
$$

that satisfies

$$
\lim _{\tilde{\mathbf{C}}_{\mathrm{in}} \rightarrow \mathbf{C}_{\mathrm{in}}} \Delta\left(\mathbf{C}_{\mathrm{in}}, \tilde{\mathbf{C}}_{\mathrm{in}}\right)=0
$$


and for any finite alphabet MAC and $\left(\mathbf{C}_{\mathrm{in}}, \tilde{\mathbf{C}}_{\mathrm{in}}, \mathbf{C}_{\mathrm{out}}\right) \in$ $\mathbb{R}_{\geq 0}^{2} \times \mathbb{R}_{\geq 0}^{2} \times \mathbb{R}_{\geq 0}^{2}$, we have

$$
\left|C_{\text {sum }}\left(\mathbf{C}_{\text {in }}, \mathbf{C}_{\text {out }}\right)-C_{\text {sum }}\left(\tilde{\mathbf{C}}_{\text {in }}, \mathbf{C}_{\text {out }}\right)\right| \leq \Delta\left(\mathbf{C}_{\text {in }}, \tilde{\mathbf{C}}_{\text {in }}\right) .
$$

We prove this lemma in Subsection IX-M. If we now apply Lemma 21 to 13 , we get

$$
\begin{aligned}
& \left|C_{\text {sum }}\left(\tilde{\mathbf{C}}_{\text {in }}, \tilde{\mathbf{C}}_{\text {out }}\right)-C_{\text {sum }}\left(\mathbf{C}_{\text {in }}, \mathbf{C}_{\text {out }}\right)\right| \\
& \leq \Delta\left(\mathbf{C}_{\text {in }}, \tilde{\mathbf{C}}_{\text {in }}\right)+\left|C_{\text {sum }}\left(\mathbf{C}_{\text {in }}, \tilde{\mathbf{C}}_{\text {out }}\right)-C_{\text {sum }}\left(\mathbf{C}_{\text {in }}, \mathbf{C}_{\text {out }}\right)\right|
\end{aligned}
$$

where $\Delta\left(\mathbf{C}_{\text {in }}, \tilde{\mathbf{C}}_{\text {in }}\right) \rightarrow 0$ whenever $\tilde{\mathbf{C}}_{\text {in }} \rightarrow \mathbf{C}_{\text {in }}$. This means that to prove the continuity of $C_{\text {sum }}\left(\tilde{\mathbf{C}}_{\text {in }}, \tilde{\mathbf{C}}_{\text {out }}\right)$ at $\left(\tilde{\mathbf{C}}_{\text {in }}, \tilde{\mathbf{C}}_{\text {out }}\right)=\left(\mathbf{C}_{\text {in }}, \mathbf{C}_{\text {out }}\right)$ it suffices to show that $C_{\text {sum }}\left(\mathbf{C}_{\text {in }}, \tilde{\mathbf{C}}_{\text {out }}\right)$ is continuous at $\tilde{\mathbf{C}}_{\text {out }}=\mathbf{C}_{\text {out }}$. We rely on this fact in the three remaining cases.

Case (b): $C_{\text {out }}^{1}=0$ and $C_{\text {out }}^{2}=0$. In this case, note that

$$
\begin{aligned}
& C_{\text {sum }}\left(\mathbf{C}_{\text {in }}, \tilde{\mathbf{C}}_{\text {out }}\right)-C_{\text {sum }}\left(\mathbf{C}_{\text {in }}, \mathbf{0}\right) \\
& \quad=C_{\text {sum }}\left(\mathbf{C}_{\text {in }}, \tilde{\mathbf{C}}_{\text {out }}\right)-C_{\text {sum }}(\mathbf{0}, \mathbf{0}) \\
& \quad \leq C_{\text {sum }}\left(\mathbf{C}_{\text {in }}^{*}, \tilde{\mathbf{C}}_{\text {out }}\right)-C_{\text {sum }}(\mathbf{0}, \mathbf{0}),
\end{aligned}
$$

where the right hand side in 14 goes to zero as $\tilde{\mathbf{C}}_{\text {out }} \rightarrow \mathbf{0}$ by Theorem 3 .

Case (c): $C_{\mathrm{in}}^{1} \geq C_{\mathrm{in}}^{1 *}$ and $C_{\mathrm{out}}^{2} \geq C_{\mathrm{out}}^{2 *}$. This case follows directly from Theorem 3

Case (d): $C_{\mathrm{in}}^{1}=0$ or $C_{\mathrm{in}}^{2}=0$. Without loss of generality, assume $C_{\text {in }}^{2}=0$. Then for any $\tilde{\mathbf{C}}_{\text {out }} \in \mathbb{R}_{\geq 0}^{2}$, we have

$$
\begin{aligned}
C_{\text {sum }}\left(\mathbf{C}_{\text {in }}, \tilde{\mathbf{C}}_{\text {out }}\right) & =C_{\text {sum }}\left(\left(C_{\text {in }}^{1}, 0\right), \tilde{\mathbf{C}}_{\text {out }}\right) \\
& =C_{\text {sum }}\left(\left(C_{\text {in }}^{1}, 0\right),\left(0, \tilde{C}_{\text {out }}^{2}\right)\right) .
\end{aligned}
$$

For a fixed $C_{\text {in }}^{1}$, let $f: \mathbb{R}_{\geq 0} \rightarrow \mathbb{R}_{\geq 0}$ denote the function

$$
f\left(C_{\text {out }}^{2}\right):=C_{\text {sum }}\left(\left(C_{\text {in }}^{1}, 0\right),\left(0, C_{\text {out }}^{2}\right)\right) .
$$

Note that $f$ is continuous on $\mathbb{R}_{>0}$ since it is concave. To prove the continuity of $f$ at $C_{\text {out }}^{2}=0$, observe that $f\left(C_{\text {out }}^{2}\right)$ equals the sum-capacity of a MAC with a $\left(C_{12}, 0\right)$-conference [4], where

$$
C_{12}:=\min \left\{C_{\text {in }}^{1}, C_{\text {out }}^{2}\right\} .
$$

From the capacity region given in [4], we have

$$
f\left(C_{\text {out }}^{2}\right) \leq f(0)+\min \left\{C_{\text {in }}^{1}, C_{\text {out }}^{2}\right\},
$$

which implies that $f$ is continuous at $C_{\text {out }}^{2}=0$. The case where $C_{\mathrm{in}}^{1}=0$ follows similarly.

\section{PROOFS}

In this section, we give detailed proofs of our results in the order they appear in the earlier sections.

\section{A. Proof of Theorem 5}

We seek to prove that for any MAC where unlimited cooperation increases sum-capacity (formally specified by the definition of $\mathcal{C}^{*}$ in Section IV], the benefit of limited-rate cooperation grows at least as quickly as the square root of the $\mathrm{CF}$ output link capacities.

Since $\mathbf{C}_{\text {in }} \in \mathbb{R}_{>0}^{2}$, there exists $\lambda=\lambda\left(\mathbf{C}_{\text {in }}\right) \in(0,1)$ such that for $i \in\{1,2\}$,

$$
C_{\mathrm{in}}^{i}>\lambda C_{\mathrm{in}}^{* i} .
$$

From the monotonicity and concavity of the average-error sum-capacity [23, Lemma 4], it follows that

$$
\begin{aligned}
C_{\text {sum }} & \left(\mathbf{C}_{\text {in }}, h \mathbf{v}\right) \\
& \geq C_{\text {sum }}\left(\lambda \mathbf{C}_{\text {in }}^{*}, \lambda h \mathbf{v}\right) \\
& \geq \lambda C_{\text {sum }}\left(\mathbf{C}_{\text {in }}^{*}, h \mathbf{v}\right)+(1-\lambda) C_{\text {sum }}(\mathbf{0}, \mathbf{0}) .
\end{aligned}
$$

Applying in (15) the fact that

$$
C_{\text {sum }}\left(\mathbf{C}_{\mathrm{in}}^{*}, \mathbf{0}\right)=C_{\text {sum }}\left(\mathbf{C}_{\mathrm{in}}, \mathbf{0}\right)=C_{\text {sum }}(\mathbf{0}, \mathbf{0})
$$

results in

$$
\begin{aligned}
& C_{\text {sum }}\left(\mathbf{C}_{\text {in }}, h \mathbf{v}\right)-C_{\text {sum }}\left(\mathbf{C}_{\text {in }}, \mathbf{0}\right) \\
& \quad \geq \lambda\left(C_{\text {sum }}\left(\mathbf{C}_{\text {in }}^{*}, h \mathbf{v}\right)-C_{\text {sum }}\left(\mathbf{C}_{\text {in }}^{*}, \mathbf{0}\right)\right) .
\end{aligned}
$$

On the other hand, setting $\mathbf{C}_{\text {out }}=h \mathbf{v}=h\left(v_{1}, v_{2}\right)$ in the lower bound of Lemma 10 gives

$$
\begin{aligned}
C_{\text {sum }}\left(\mathbf{C}_{\mathrm{in}}^{*}, h \mathbf{v}\right) & \geq \sigma\left(h\left(v_{1}+v_{2}\right)\right)-\min \left\{h v_{1}, h v_{2}\right\} \\
& \geq \sigma_{1}\left(h\left(v_{1}+v_{2}\right)\right)-\min \left\{h v_{1}, h v_{2}\right\},
\end{aligned}
$$

where $\sigma$ and $\sigma_{1}$ are defined in (9) and (11), respectively, and (17) follows from (9). Note that

$$
\sigma_{1}(0)=C_{\text {sum }}(\mathbf{0}, \mathbf{0})=C_{\text {sum }}\left(\mathbf{C}_{\mathrm{in}}^{*}, \mathbf{0}\right) .
$$

Therefore, combining (16) and (17) gives

$$
\begin{aligned}
& C_{\text {sum }}\left(\mathbf{C}_{\text {in }}, h \mathbf{v}\right)-C_{\text {sum }}\left(\mathbf{C}_{\mathrm{in}}, \mathbf{0}\right) \\
& \quad \geq \lambda\left(\sigma_{1}\left(h v_{1}+h v_{2}\right)-\sigma_{1}(0)\right)-\lambda h \min \left\{v_{1}, v_{2}\right\} .
\end{aligned}
$$

Given (18), the following lemma completes the proof.

Lemma 22. For any discrete memoryless $M A C$ in $\mathcal{C}^{*}$, we have

$$
\liminf _{\delta \rightarrow 0^{+}} \frac{\sigma_{1}(\delta)-\sigma_{1}(0)}{\sqrt{\delta}}>0 .
$$

The key result employed in proving Lemma 22 is the asymptotic equivalence of the Kullback-Liebler (KL) and the chi-squared divergences [24, Theorem 4.1, p. 448], which we next describe. Let $p(x)$ and $q(x)$ be distributions on a finite alphabet $\mathcal{X}$. Then the KL and the chi-squared divergences between $p$ and $q$ are given by

$$
\begin{aligned}
D(p(x) \| q(x)) & :=\sum_{x \in \mathcal{X}} p(x) \log \frac{p(x)}{q(x)} \\
\chi^{2}(p(x), q(x)) & :=\sum_{x \in \mathcal{X}} \frac{(p(x)-q(x))^{2}}{q(x)},
\end{aligned}
$$

respectively. Suppose $q$ has full support; that is, for all $x \in \mathcal{X}$, $q(x)>0$. Then if $p$ tends to $q$ pointwise on $\mathcal{X}$, then

$$
\frac{D(p(x) \| q(x))}{\chi^{2}(p(x), q(x))} \rightarrow \frac{1}{2 \ln 2} .
$$

If additional assumptions are made regarding how $p$ tends to $q$ in (19), one can make somewhat stronger statements regarding the connection between the $\mathrm{KL}$ and chi-squared divergences. Details appear in Lemma 23, below. Similar ideas also appear in other recent work, in particular in the field of covert communication [25], [26]. 
Lemma 23. Let $\mathcal{X}$ be a finite set. Consider distributions $p_{0}$ and $p_{1}$ on $\mathcal{X}$ and assume that the support of $p_{1}$ is contained in that of $p_{0}$. For each $\lambda \in(0,1)$, define the distribution $p_{\lambda}$ on $\mathcal{X}$ as

$$
p_{\lambda}(x):=(1-\lambda) p_{0}(x)+\lambda p_{1}(x) .
$$

Then the following statements hold:

(a) In the neighborhood of $\lambda=0^{+}$,

$$
D\left(p_{\lambda}(x) \| p_{0}(x)\right)=K_{0} \lambda^{2} \chi^{2}\left(p_{1}(x), p_{0}(x)\right)+o\left(\lambda^{2}\right),
$$

where $K_{0}:=\frac{1}{2 \ln 2}$.

(b) The mapping

$$
\lambda \mapsto \frac{d}{d \lambda} D\left(p_{\lambda}(x) \| p_{0}(x)\right),
$$

is well-defined and continuous on $[0,1)$; that is, the mapping

$$
\lambda \mapsto D\left(p_{\lambda}(x) \| p_{0}(x)\right)
$$

is continuously differentiable on $[0,1)$.

Proof of Lemma 23. For Part (a), combining the fact that

$$
\chi^{2}\left(p_{\lambda}(x), p_{0}(x)\right)=\lambda^{2} \chi^{2}\left(p_{1}(x), p_{0}(x)\right),
$$

with (19) directly implies (20).

For Part (b), let $\mathcal{S}_{\lambda} \subseteq \mathcal{X}$ be the support of $p_{\lambda}(x)$. Note that $\mathcal{S}_{1} \subseteq \mathcal{S}_{0}$ (which holds by assumption) implies that $\mathcal{S}_{\lambda}=\mathcal{S}_{0}$ for all $\lambda \in[0,1)$. Thus we can write

$$
D\left(p_{\lambda}(x) \| p_{0}(x)\right)=\sum_{x \in \mathcal{S}_{0}} p_{\lambda}(x) \log \frac{p_{\lambda}(x)}{p_{0}(x)} .
$$

If we differentiate both sides of 22 with respect to $\lambda$, we get

$$
\frac{d}{d \lambda} D\left(p_{\lambda}(x) \| p_{0}(x)\right)=\sum_{x \in \mathcal{S}_{0}}\left(p_{1}(x)-p_{0}(x)\right) \log \frac{p_{\lambda}(x)}{p_{0}(x)} .
$$

Thus for the continuity of (21), it suffices to consider the continuity of the mapping

$$
\lambda \mapsto \log \frac{p_{\lambda}(x)}{p_{0}(x)}
$$

over $\lambda \in[0,1)$ for each $x \in \mathcal{S}_{0}$. Fix one such $x$, say $x=x_{0}$. Then since (i) $p_{\lambda}\left(x_{0}\right)$ is continuous in $\lambda$, (ii) for any $\lambda \in$ $[0,1), p_{\lambda}\left(x_{0}\right)$ is positive, and (iii) $t \mapsto \log (t)$ is continuous on $(0, \infty)$, we conclude that 24$)$ is continuous on $[0,1)$ as well.

Using Lemma 23, we next prove Lemma 22, which consequently proves Theorem 1 .

Proof of Lemma 22. Recall from (11) that $\sigma_{1}$ is given by

$$
\sigma_{1}(\delta)=\sup _{\mathcal{U}} \max _{p \in \mathcal{P}_{\mathcal{U}}^{(1)}(\delta)} I\left(X_{1}, X_{2} ; Y \mid U\right),
$$

where the supremum is over all finite sets $\mathcal{U}$. Thus we can bound $\sigma_{1}$ from below by

$$
\sigma_{1}(\delta) \geq \max _{p \in \mathcal{P}^{(1)}(\delta)} I\left(X_{1}, X_{2} ; Y\right),
$$

where $\mathcal{P}^{(1)}(\delta)$ is simply the set of all distributions $p\left(x_{1}, x_{2}\right)$ that satisfy $I\left(X_{1} ; X_{2}\right) \leq \delta$. In what follows, we use 25 to prove the desired result. This amounts to constructing a family of distributions $\left(q_{\delta}\left(x_{1}, x_{2}\right)\right)_{\delta}$ where (i) each $q_{\delta} \in \mathcal{P}^{(1)}(\delta)$, (ii) the mapping

$$
\sigma_{L B}(\delta):=\left.I\left(X_{1}, X_{2} ; Y\right)\right|_{p\left(x_{1}, x_{2}\right)=q_{\delta}\left(x_{1}, x_{2}\right)}
$$

satisfies

$$
\lim _{\delta \rightarrow 0^{+}} \frac{\sigma_{L B}(\delta)-\sigma_{L B}(0)}{\sqrt{\delta}}>0,
$$

and (iii) $\sigma_{L B}(0)=\sigma_{1}(0)$. The ' $L B$ ' in $\sigma_{L B}$ signifies the fact that $\sigma_{L B}$ is a lower bound for $\sigma_{1}$ by (25).

Since our MAC is in $\mathcal{C}^{*}$, by definition, there exists a distribution $p_{0}\left(x_{1}\right) p_{0}\left(x_{2}\right)$ with support $\mathcal{S}_{0} \subseteq \mathcal{X}_{1} \times \mathcal{X}_{2}$ that satisfies

$$
I_{0}\left(X_{1}, X_{2} ; Y\right)=\max _{p\left(x_{1}\right) p\left(x_{2}\right)} I\left(X_{1}, X_{2} ; Y\right),
$$

and a distribution $p_{1}\left(x_{1}, x_{2}\right)$ with support $\mathcal{S}_{1} \subseteq \mathcal{S}_{0}$ that satisfies

$$
I_{1}\left(X_{1}, X_{2} ; Y\right)+D\left(p_{1}(y) \| p_{0}(y)\right)>I_{0}\left(X_{1}, X_{2} ; Y\right) .
$$

For each $\lambda \in(0,1)$, define the distribution $p_{\lambda}\left(x_{1}, x_{2}\right)$ as

$$
p_{\lambda}\left(x_{1}, x_{2}\right):=(1-\lambda) p_{0}\left(x_{1}\right) p_{0}\left(x_{2}\right)+\lambda p_{1}\left(x_{1}, x_{2}\right) .
$$

Let $\mathcal{S}_{\lambda} \subseteq \mathcal{X}_{1} \times \mathcal{X}_{2}$ denote the support of $p_{\lambda}\left(x_{1}, x_{2}\right)$. Then for $\lambda \in[0,1)$, we have $\mathcal{S}_{\lambda}=\mathcal{S}_{0}$, since by assumption, $\mathcal{S}_{1} \subseteq \mathcal{S}_{0}$. Furthermore, let $\mathcal{S}_{\lambda}^{(1)}$ and $\mathcal{S}_{\lambda}^{(2)}$ denote the projections of $\mathcal{S}_{\lambda}$ on $\mathcal{X}_{1}$ and $\mathcal{X}_{2}$, respectively. Based on this notation, we can write

$$
\begin{aligned}
I_{\lambda}\left(X_{1} ;\right. & \left.X_{2}\right) \\
= & \sum_{\left(x_{1}, x_{2}\right) \in \mathcal{S}_{0}} p_{\lambda}\left(x_{1}, x_{2}\right) \log \frac{p_{\lambda}\left(x_{1}, x_{2}\right)}{p_{\lambda}\left(x_{1}\right) p_{\lambda}\left(x_{2}\right)} \\
= & \sum_{\left(x_{1}, x_{2}\right) \in \mathcal{S}_{0}} p_{\lambda}\left(x_{1}, x_{2}\right) \log \frac{p_{\lambda}\left(x_{1}, x_{2}\right)}{p_{0}\left(x_{1}\right) p_{0}\left(x_{2}\right)} \\
& +\sum_{\left(x_{1}, x_{2}\right) \in \mathcal{S}_{0}} p_{\lambda}\left(x_{1}, x_{2}\right) \log \frac{p_{0}\left(x_{1}\right) p_{0}\left(x_{2}\right)}{p_{\lambda}\left(x_{1}\right) p_{\lambda}\left(x_{2}\right)} \\
= & \sum_{\left(x_{1}, x_{2}\right) \in \mathcal{S}_{0}} p_{\lambda}\left(x_{1}, x_{2}\right) \log \frac{p_{\lambda}\left(x_{1}, x_{2}\right)}{p_{0}\left(x_{1}\right) p_{0}\left(x_{2}\right)} \\
& +\sum_{x_{1} \in \mathcal{S}_{0}^{(1)}} p_{\lambda}\left(x_{1}\right) \log \frac{p_{0}\left(x_{1}\right)}{p_{\lambda}\left(x_{1}\right)} \\
& +\sum_{x_{2} \in \mathcal{S}_{0}^{(2)}} p_{\lambda}\left(x_{2}\right) \log \frac{p_{0}\left(x_{2}\right)}{p_{\lambda}\left(x_{2}\right)} \\
= & D\left(p_{\lambda}\left(x_{1}, x_{2}\right) \| p_{0}\left(x_{1}\right) p_{0}\left(x_{2}\right)\right)-D\left(p_{\lambda}\left(x_{1}\right) \| p_{0}\left(x_{1}\right)\right) \\
& -D\left(p_{\lambda}\left(x_{2}\right) \| p_{0}\left(x_{2}\right)\right)
\end{aligned}
$$

If we now apply Part (a) of Lemma 23 to 28 , we get

$$
\lim _{\lambda \rightarrow 0^{+}} \frac{1}{\lambda^{2}} I_{\lambda}\left(X_{1} ; X_{2}\right)=K_{1},
$$

where

$$
\begin{aligned}
K_{1}:= & K_{0}\left(\chi^{2}\left(p_{1}\left(x_{1}, x_{2}\right), p_{0}\left(x_{1}\right) p_{0}\left(x_{2}\right)\right)\right. \\
& \left.-\chi^{2}\left(p_{1}\left(x_{1}\right), p_{0}\left(x_{1}\right)\right)-\chi^{2}\left(p_{1}\left(x_{2}\right), p_{0}\left(x_{2}\right)\right)\right) .
\end{aligned}
$$


Since $\mathcal{S}_{1} \subseteq \mathcal{S}_{0}$, all chi-squared distances in 30 are welldefined. Also, since mutual information is always nonnegative, by (29) we have $K_{1} \geq 0$. In the discussion that follows, we seek to understand the relationship between the "cooperation rate," $I_{\lambda}\left(X_{1} ; X_{2}\right)$, and $\lambda$, the "time-sharing coefficient." To this end, fix $\epsilon>0$, and define the mapping $\delta^{*}:[0,1] \rightarrow \mathbb{R}$ as

$$
\delta^{*}(\theta):=I_{\sqrt{\theta}}\left(X_{1} ; X_{2}\right)+\epsilon \theta .
$$

Here $\theta$ acts as an auxiliary variable in lieu of $\lambda$; its purpose is to allow the application of the inverse function theorem. Prior to that application however, we highlight some of the properties of the function $\delta^{*}$.

First, note that for all $\theta \in[0,1]$,

$$
I_{\sqrt{\theta}}\left(X_{1} ; X_{2}\right) \leq \delta^{*}(\theta) .
$$

Second, by 29), we have

$$
\left.\frac{d \delta^{*}}{d \theta}\right|_{\theta=0^{+}}=\lim _{\theta \rightarrow 0^{+}} \frac{\delta^{*}(\theta)}{\theta}=K_{1}+\epsilon>0 .
$$

Intuitively, (31) and (32) characterize the behavior of $\delta$ as a function of the auxiliary variable $\theta=\lambda^{2}$. However, towards our goal of constructing the family of distributions $\left(q_{\delta}\left(x_{1}, x_{2}\right)\right)_{\delta}$ we require the inverse relation; that is, we require $\lambda$ as a function of $\delta$. For this purpose, we apply the inverse function theorem below.

First, Part (b) of Lemma 23, together with 28, implies that $\delta^{*}$ is continuously differentiable on $[0,1)$. Second, from 32 , $\delta^{*}$ has nonzero derivative at $\theta=0^{+}$. Therefore, we can apply the inverse function theorem [27, p. 289, Theorem 23] which implies the following statements.

(i) There exists $\theta_{0}>0$ and $\delta_{0}>0$ such that $\delta^{*}$ maps $\left[0, \theta_{0}\right)$ to $\left[0, \delta_{0}\right)$ bijectively.

(ii) The inverse of $\delta^{*}$ on $\left[0, \theta_{0}\right)$, which we denote by $\theta^{*}:\left[0, \delta_{0}\right) \rightarrow\left[0, \theta_{0}\right)$, is continuously differentiable.

(iii) The inverse relation of 32 holds; that is,

$$
\begin{aligned}
\lim _{\delta \rightarrow 0^{+}} \frac{\theta^{*}(\delta)}{\delta} & =\left.\frac{d \theta^{*}}{d \delta}\right|_{\delta^{+}=0} \\
& =\left(\left.\frac{d \delta^{*}}{d \theta}\right|_{\theta=0^{+}}\right)^{-1}=\frac{1}{K_{1}+\epsilon} .
\end{aligned}
$$

Next, define the mapping $\lambda^{*}:\left[0, \delta_{0}\right) \rightarrow[0,1]$ as $\lambda^{*}(\delta):=$ $\sqrt{\theta^{*}(\delta)}$. From (i) it follows that $\lambda^{*}(0)=0$, while 31 implies

$$
I_{\lambda^{*}(\delta)}\left(X_{1} ; X_{2}\right) \leq \delta
$$

Next, rewriting 33 in terms of $\lambda^{*}(\delta)$ gives

$$
\lim _{\delta \rightarrow 0^{+}} \frac{\lambda^{*}(\delta)}{\sqrt{\delta}}=\frac{1}{\sqrt{K_{1}+\epsilon}}
$$

Thus we have

$$
\lambda^{*}(\delta)=\sqrt{\frac{\delta}{K_{1}+\epsilon}}+o(\sqrt{\delta}) .
$$

As we show below, a suitable choice for the family of distributions $\left(q_{\delta}\left(x_{1}, x_{2}\right)\right)_{\delta}$ is to set, for each $\delta \in\left[0, \delta_{0}\right)$ :

$$
q_{\delta}\left(x_{1}, x_{2}\right):=p_{\lambda^{*}(\delta)}\left(x_{1}, x_{2}\right) .
$$

Now for $\lambda \in[0,1)$, consider $I_{\lambda}\left(X_{1}, X_{2} ; Y\right)$, the mutual information between $\left(X_{1}, X_{2}\right)$ and $Y$, computed with respect to the distribution $p_{\lambda}\left(x_{1}, x_{2}\right) p\left(y \mid x_{1}, x_{2}\right)$. Direct differentiation gives [6. Lemma 15, Part (iii)]

$$
\begin{aligned}
I_{\lambda}\left(X_{1},\right. & \left.X_{2} ; Y\right) \\
= & I_{0}\left(X_{1}, X_{2} ; Y\right)+\left(I_{1}\left(X_{1}, X_{2} ; Y\right)-I_{0}\left(X_{1}, X_{2} ; Y\right)\right. \\
& \left.+D\left(p_{1}(y) \| p_{0}(y)\right)\right) \lambda+o(\lambda) .
\end{aligned}
$$

If we now set $\lambda=\lambda^{*}(\delta)$, define the constant $K$ as

$$
\begin{aligned}
K:=\frac{1}{\sqrt{K_{1}+\epsilon}}( & I_{1}\left(X_{1}, X_{2} ; Y\right)-I_{0}\left(X_{1}, X_{2} ; Y\right) \\
& \left.+D\left(p_{1}(y) \| p_{0}(y)\right)\right),
\end{aligned}
$$

and apply $[36$, we get

$$
I_{\lambda^{*}(\delta)}\left(X_{1}, X_{2} ; Y\right)=I_{0}\left(X_{1}, X_{2} ; Y\right)+K \sqrt{\delta}+o(\sqrt{\delta}),
$$

where $K>0$ by [27]. Note that by 34] and 25],

$$
\sigma_{1}(\delta) \geq I_{\lambda^{*}(\delta)}\left(X_{1}, X_{2} ; Y\right) .
$$

Furthermore, since by (26), we have

$$
\sigma_{1}(0)=\max _{p\left(x_{1}\right) p\left(x_{2}\right)} I\left(X_{1}, X_{2} ; Y\right)=I_{0}\left(X_{1}, X_{2} ; Y\right),
$$

and $\lambda^{*}(0)=0$, combining 37 and 38 gives

$$
\begin{aligned}
& \liminf _{\delta \rightarrow 0^{+}} \frac{\sigma_{1}(\delta)-\sigma_{1}(0)}{\sqrt{\delta}} \\
& \quad \geq \lim _{\delta \rightarrow 0^{+}} \frac{I_{\lambda^{*}(\delta)}\left(X_{1}, X_{2} ; Y\right)-I_{0}\left(X_{1}, X_{2} ; Y\right)}{\sqrt{\delta}}=K>0 .
\end{aligned}
$$

This concludes the proof of Lemma 22 .

\section{B. Proof of Corollary 7}

The discussion following Theorem 6, together with Lemma 21. implies that it suffices to show that for any $\left(\mathbf{C}_{\text {in }}, \mathbf{C}_{\text {out }}\right)$ where $C_{\text {out }}^{2}=0$ (or by symmetry, the corresponding equation for $C_{\text {out }}^{1}=0$ ),

$$
\lim _{\tilde{\mathbf{C}}_{\text {out }} \rightarrow \mathbf{C}_{\text {out }}} C_{\text {sum }}\left(\mathbf{C}_{\text {in }},\left(\tilde{C}_{\text {out }}^{1}, \tilde{C}_{\text {out }}^{2}\right)\right)=C_{\text {sum }}\left(\mathbf{C}_{\text {in }},\left(C_{\text {out }}^{1}, 0\right)\right)
$$

is equivalent to

$$
\lim _{\tilde{C}_{\text {out }}^{2} \rightarrow 0^{+}} C_{\text {sum }}\left(\mathbf{C}_{\text {in }},\left(C_{\text {out }}^{1}, \tilde{C}_{\text {out }}^{2}\right)\right)=C_{\text {sum }}\left(\mathbf{C}_{\text {in }},\left(C_{\text {out }}^{1}, 0\right)\right) .
$$

To prove this, we require the next lemma for concave functions that are nondecreasing as well. We prove this lemma in Subsection IX-N.

Lemma 24. Let $f: \mathbb{R}_{\geq 0} \rightarrow \mathbb{R}_{\geq 0}$ be concave and nondecreasing. Then if $|x-y| \leq \min \{x, y\}$,

$$
|f(x)-f(y)| \leq f(|x-y|)-f(0) .
$$

We have

$$
\begin{aligned}
& \left|C_{\text {sum }}\left(\mathbf{C}_{\text {in }},\left(\tilde{C}_{\text {out }}^{1}, \tilde{C}_{\text {out }}^{2}\right)\right)-C_{\text {sum }}\left(\mathbf{C}_{\text {in }},\left(C_{\text {out }}^{1}, 0\right)\right)\right| \\
& \quad \stackrel{(a)}{\leq}\left|C_{\text {sum }}\left(\mathbf{C}_{\text {in }},\left(\tilde{C}_{\text {out }}^{1}, \tilde{C}_{\text {out }}^{2}\right)\right)-C_{\text {sum }}\left(\mathbf{C}_{\text {in }},\left(C_{\text {out }}^{1}, \tilde{C}_{\text {out }}^{2}\right)\right)\right| \\
& \quad+\left|C_{\text {sum }}\left(\mathbf{C}_{\text {in }},\left(C_{\text {out }}^{1}, \tilde{C}_{\text {out }}^{2}\right)\right)-C_{\text {sum }}\left(\mathbf{C}_{\text {in }},\left(C_{\text {out }}^{1}, 0\right)\right)\right| \\
& \quad \stackrel{(b)}{\leq} \mid C_{\text {sum }}\left(\mathbf{C}_{\text {in }},\left(\left|\tilde{C}_{\text {out }}^{1}-C_{\text {out }}^{1}\right|, \tilde{C}_{\text {out }}^{2}\right)\right) \\
& \quad-C_{\text {sum }}\left(\mathbf{C}_{\text {in }},\left(0, \tilde{C}_{\text {out }}^{2}\right)\right) \mid \\
& \quad+\left|C_{\text {sum }}\left(\mathbf{C}_{\text {in }},\left(C_{\text {out }}^{1}, \tilde{C}_{\text {out }}^{2}\right)\right)-C_{\text {sum }}\left(\mathbf{C}_{\text {in }},\left(C_{\text {out }}^{1}, 0\right)\right)\right|,
\end{aligned}
$$


where (a) follows from the triangle inequality, and (b) follows from applying Lemma 24 to the mapping

$$
\tilde{C}_{\text {out }}^{1} \mapsto C_{\text {sum }}\left(\mathbf{C}_{\text {in }},\left(\tilde{C}_{\text {out }}^{1}, \tilde{C}_{\text {out }}^{2}\right)\right) .
$$

If we now let $\tilde{\mathbf{C}}_{\text {out }} \rightarrow\left(C_{\text {out }}^{1}, 0\right)$, Case (b) of Theorem 6 , together with another application of the triangle inequality, implies that

$$
\begin{gathered}
\lim _{\tilde{\mathbf{C}}_{\text {out }} \rightarrow\left(C_{\text {out }}^{1}, 0\right)} \mid C_{\text {sum }}\left(\mathbf{C}_{\text {in }},\left(\left|\tilde{C}_{\text {out }}^{1}-C_{\text {out }}^{1}\right|, \tilde{C}_{\text {out }}^{2}\right)\right) \\
-C_{\text {sum }}\left(\mathbf{C}_{\text {in }},\left(0, \tilde{C}_{\text {out }}^{2}\right)\right) \mid=0
\end{gathered}
$$

from which our result follows.

\section{Proof of Theorem 8}

By Proposition 2, we only need to prove one direction. Specifically, here we show that for a fixed MAC and $\mathbf{C}_{\text {in }} \in$ $\mathbb{R}_{>0}^{2}$, if $C_{\text {sum, } \max }\left(\mathbf{C}_{\text {in }}, \mathbf{C}_{\text {out }}\right)$ is not continuous at $\mathbf{C}_{\text {out }}=\mathbf{0}$, then

$$
C_{\text {sum }}(\mathbf{0}, \mathbf{0})>C_{\text {sum, } \max }(\mathbf{0}, \mathbf{0}) .
$$

This is equivalent to showing that if

$$
C_{\text {sum }}(\mathbf{0}, \mathbf{0})=C_{\text {sum }, \max }(\mathbf{0}, \mathbf{0}),
$$

then $C_{\text {sum, } \max }\left(\mathbf{C}_{\text {in }}, \mathbf{C}_{\text {out }}\right)$ is continuous at $\mathbf{C}_{\text {out }}=\mathbf{0}$.

We begin by defining the function $f: \mathbb{R}_{\geq 0} \rightarrow \mathbb{R}_{\geq 0}$ as

$$
f\left(C_{\text {out }}\right):=C_{\text {sum, max }}\left(\mathbf{C}_{\text {in }}^{*},\left(C_{\text {out }}, C_{\text {out }}\right)\right) \text {, }
$$

where $\mathbf{C}_{\mathrm{in}}^{*}$ is defined in Section $\mathrm{V}$. Then by [5, Theorem 1] for all $C_{\text {out }}>0$, and by $(40)$ for $C_{\text {out }}=0$, we have

$$
f\left(C_{\text {out }}\right)=C_{\text {sum }}\left(\mathbf{C}_{\text {in }}^{*},\left(C_{\text {out }}, C_{\text {out }}\right)\right) .
$$

For each $\mathbf{C}_{\text {out }} \in \mathbb{R}_{\geq 0}^{2}$, let $C_{\text {out }}^{*}:=\max \left\{C_{\text {out }}^{1}, C_{\text {out }}^{2}\right\}$. Then for any $\mathbf{C}_{\text {in }} \in \mathbb{R}_{\geq 0}^{2}$,

$$
f(0) \leq C_{\text {sum, } \max }\left(\mathbf{C}_{\text {in }}, \mathbf{C}_{\text {out }}\right) \leq f\left(C_{\text {out }}^{*}\right) .
$$

If we now let $\mathbf{C}_{\text {out }} \rightarrow \mathbf{0}$ and apply Theorem 3 , the continuity of $C_{\text {sum, } \max }\left(\mathbf{C}_{\text {in }}, \mathbf{C}_{\text {out }}\right)$ at $\mathbf{C}_{\text {out }}=\mathbf{0}$ follows.

\section{Proof of Lemma 9}

Our goal here is to show that for integers $m, n \geq 1$ and $\delta \geq 0$,

$$
(m+n) \sigma_{m+n}(\delta) \geq m \sigma_{m}(\delta)+n \sigma_{n}(\delta) .
$$

By the definition of $\sigma_{n}(\delta)$, for all $\epsilon>0$, there exist finite alphabets $\mathcal{U}_{0}$ and $\mathcal{U}_{1}$ and distributions $p_{n} \in \mathcal{P}_{\mathcal{U}_{0}}^{(n)}(\delta)$ and $p_{m} \in$ $\mathcal{P}_{\mathcal{U}_{1}}^{(m)}(\delta)$ such that

$$
\begin{aligned}
I_{n}\left(X_{1}^{n}, X_{2}^{n} ; Y^{n} \mid U_{0}\right) & \geq n \sigma_{n}(\delta)-n \epsilon \\
I_{m}\left(X_{1}^{m}, X_{2}^{m} ; Y^{m} \mid U_{1}\right) & \geq m \sigma_{m}(\delta)-m \epsilon .
\end{aligned}
$$

Consider the distribution

$$
\begin{aligned}
p_{n+m} & \left(u_{0}, u_{1}, x_{1}^{n+m}, x_{2}^{n+m}\right) \\
& :=p_{n}\left(u_{0}, x_{1}^{n}, x_{2}^{n}\right) p_{m}\left(u_{1}, x_{1}^{n+1: n+m}, x_{2}^{n+1: n+m}\right) .
\end{aligned}
$$

Let $\mathcal{U}:=\mathcal{U}_{0} \times \mathcal{U}_{1}$. Then it is straightforward to show that $p_{n+m} \in \mathcal{P}_{\mathcal{U}}^{(n+m)}(\delta)$, and

$$
\begin{aligned}
& I_{n+m}\left(X_{1}^{n+m}, X_{2}^{n+m} ; Y^{n+m} \mid U_{0}, U_{1}\right) \\
& \quad \geq n \sigma_{n}(\delta)+m \sigma_{m}(\delta)-(n+m) \epsilon,
\end{aligned}
$$

which implies the desired result.

\section{E. Proof of Lemma 10}

Here we prove lower and upper bounds for $C_{\text {sum }}\left(\mathbf{C}_{\text {in }}^{*}, \mathbf{C}_{\text {out }}\right)$ in terms of $\sigma\left(C_{\text {out }}^{1}+C_{\text {out }}^{2}\right)$.

The lower bound follows directly from [6]. Specifically, for a positive integer $n$, if we apply [6, Corollary 8] to the MAC

$$
p\left(y^{n} \mid x_{1}^{n}, x_{2}^{n}\right)=\prod_{t \in[n]} p\left(y_{t} \mid x_{1 t}, x_{2 t}\right),
$$

we find that for any finite alphabet $\mathcal{U}$ and any distribution $p\left(u, x_{1}^{n}, x_{2}^{n}\right)$ that satisfies

$$
I\left(X_{1}^{n} ; X_{2}^{n} \mid U\right) \leq n\left(C_{\text {out }}^{1}+C_{\text {out }}^{2}\right),
$$

it holds that

$$
\begin{aligned}
& n C_{\text {sum }}\left(\mathbf{C}_{\text {in }}^{*}, \mathbf{C}_{\text {out }}\right) \\
& \quad \geq I\left(X_{1}^{n}, X_{2}^{n} ; Y^{n} \mid U\right)-n \min \left\{C_{\text {out }}^{1}, C_{\text {out }}^{2}\right\} .
\end{aligned}
$$

This completes the proof of the lower bound.

For the upper bound, consider a sequence of $\left(2^{n R_{1}}, 2^{n R_{2}}, n\right)$-codes for the MAC with a $\left(\mathbf{C}_{\text {in }}^{*}, \mathbf{C}_{\text {out }}\right)$ CF. For a fixed positive integer $n$, let $W_{1}$ and $W_{2}$ be independent random variables distributed uniformly on $\left[2^{n R_{1}}\right]$ and $\left[2^{n R_{2}}\right]$, respectively. Furthermore, for the blocklength- $n$ code and transmitted message pair $\left(W_{1}, W_{2}\right)$, let random variables $Z_{1} \in\left[2^{n C_{\text {out }}^{1}}\right]$ and $Z_{2} \in\left[2^{n C_{\text {out }}^{2}}\right]$ denote the information the $\mathrm{CF}$ sends to encoders 1 and 2 respectively. Note that $\left(Z_{1}, Z_{2}\right)$ is a deterministic function of $\left(W_{1}, W_{2}\right)$. By the data processing inequality,

$$
\begin{aligned}
I\left(X_{1}^{n} ; X_{2}^{n}\right) \leq & I\left(X_{1}^{n} ; W_{2}, Z_{2}\right) \\
\leq & I\left(W_{1}, Z_{1} ; W_{2}, Z_{2}\right) \\
= & H\left(W_{1}, Z_{1}\right)+H\left(W_{2}, Z_{2}\right) \\
& -H\left(W_{1}, W_{2}, Z_{1}, Z_{2}\right) \\
= & H\left(Z_{1} \mid W_{1}\right)+H\left(Z_{2} \mid W_{2}\right) \\
\leq & n\left(C_{\text {out }}^{1}+C_{\text {out }}^{2}\right) .
\end{aligned}
$$

In addition, from Fano's inequality it follows that there exists a sequence $\left(\epsilon_{n}\right)_{n=1}^{\infty}$ such that

$$
H\left(W_{1}, W_{2} \mid Y^{n}\right) \leq n \epsilon_{n},
$$

and $\epsilon_{n} \rightarrow 0$ as $n \rightarrow \infty$. We have

$$
\begin{aligned}
n\left(R_{1}+R_{2}\right) & =H\left(W_{1}, W_{2}\right) \\
& =I\left(W_{1}, W_{2} ; Y^{n}\right)+H\left(W_{1}, W_{2} \mid Y^{n}\right) \\
& \leq I\left(W_{1}, W_{2} ; Y^{n}\right)+n \epsilon_{n} \\
& =I\left(X_{1}^{n}, X_{2}^{n} ; Y^{n}\right)+n \epsilon_{n} \\
& \leq n \sigma\left(C_{\text {out }}^{1}+C_{\text {out }}^{2}\right)+n \epsilon_{n} .
\end{aligned}
$$

Dividing by $n$ and taking the limit as $n \rightarrow \infty$ completes the proof.

\section{F. Proof of Lemma 12}

For $n \geq 1$, we use the auxiliary random variable $U$ in the definition of $\sigma_{n}$ to show that $\sigma_{n}$ is concave. 
It suffices to prove the result for $n=1$ since the proof for arbitrary positive integers follows similarly. We apply the technique from [22, Appendix B]. Recall that

$$
\sigma_{1}(\delta)=\sup _{\mathcal{U}} \max _{p \in \mathcal{P}_{\mathcal{U}}^{(1)}(\delta)} I\left(X_{1}, X_{2} ; Y \mid U\right)
$$

Fix $a, b \geq 0, \lambda \in(0,1)$, and $\epsilon>0$. By definition, there exist finite sets $\mathcal{U}_{0}$ and $\mathcal{U}_{1}$ and distributions $p_{0} \in \mathcal{P}_{\mathcal{U}_{0}}^{(1)}(a)$ and $p_{1} \in \mathcal{P}_{\mathcal{U}_{1}}^{(1)}(b)$ satisfying

$$
\begin{aligned}
& I_{0}\left(X_{1}, X_{2} ; Y \mid U_{0}\right) \geq \sigma_{1}(a)-\epsilon \\
& I_{1}\left(X_{1}, X_{2} ; Y \mid U_{1}\right) \geq \sigma_{1}(b)-\epsilon,
\end{aligned}
$$

respectively. Define the alphabet $\mathcal{V}$ as

$$
\mathcal{V}:=\left(\{0\} \times \mathcal{U}_{0}\right) \cup\left(\{1\} \times \mathcal{U}_{1}\right) .
$$

We denote an element of $\mathcal{V}$ by $v=\left(v_{1}, v_{2}\right)$. Define the distribution $p_{\lambda}\left(v, x_{1}, x_{2}\right)$ as

$$
p_{\lambda}\left(v, x_{1}, x_{2}\right)=p_{\lambda}\left(v_{1}\right) p_{v_{1}}\left(v_{2}, x_{1}, x_{2}\right),
$$

where

$$
p_{\lambda}\left(v_{1}\right)= \begin{cases}1-\lambda & \text { if } v_{1}=0 \\ \lambda & \text { if } v_{1}=1\end{cases}
$$

Then for the mutual information $I_{\lambda}\left(X_{1} ; X_{2} \mid V\right)$ computed with respect to $p_{\lambda}\left(v, x_{1}, x_{2}\right)$ we have

$$
\begin{aligned}
I_{\lambda}\left(X_{1} ; X_{2} \mid V\right) \\
= \\
=I_{\lambda}\left(X_{1}, X_{2} \mid V_{1}, V_{2}\right) \\
=(1-\lambda) I\left(X_{1} ; X_{2} \mid V_{1}=0, V_{2}\right) \\
\quad+\lambda I\left(X_{1} ; X_{2} \mid V_{1}=1, V_{2}\right) \\
=(1-\lambda) I_{0}\left(X_{1} ; X_{2} \mid U_{0}\right)+\lambda I_{1}\left(X_{1} ; X_{2} \mid U_{1}\right) \\
\leq(1-\lambda) a+\lambda b,
\end{aligned}
$$

which implies $p_{\lambda} \in \mathcal{P}_{\mathcal{V}}^{(1)}((1-\lambda) a+\lambda b)$. Similarly,

$$
\begin{aligned}
I_{\lambda}\left(X_{1},\right. & \left.X_{2} ; Y \mid V\right) \\
= & I_{\lambda}\left(X_{1}, X_{2} ; Y \mid V_{1}, V_{2}\right) \\
= & (1-\lambda) I\left(X_{1}, X_{2} ; Y \mid V_{1}=0, V_{2}\right) \\
& +\lambda I\left(X_{1}, X_{2} ; Y \mid V_{1}=1, V_{2}\right) \\
= & (1-\lambda) I_{0}\left(X_{1}, X_{2} ; Y \mid U_{0}\right)+\lambda I_{1}\left(X_{1}, X_{2} ; Y \mid U_{1}\right) \\
\geq & (1-\lambda) \sigma_{1}(a)+\lambda \sigma_{1}(b)-\epsilon .
\end{aligned}
$$

Therefore,

$$
\sigma_{1}((1-\lambda) a+\lambda b) \geq(1-\lambda) \sigma_{1}(a)+\lambda \sigma_{1}(b)-\epsilon .
$$

The result now follows from the fact that the above equation holds for all $\epsilon>0$.

\section{G. Proof of Lemma 13 (Dueck's Lemma)}

This proof originally appears in [19, Subsection II.D]. We provide it here for completeness.

If for all $t \in[n]$, we have

$$
I\left(X_{1 t} ; X_{2 t} \mid U\right) \leq \epsilon,
$$

then we define $T:=\varnothing$. Otherwise, we put off the definition of $T$, noting that there exists $t_{1} \in[n]$ such that

$$
I\left(X_{1 t_{1}} ; X_{2 t_{1}} \mid U\right)>\epsilon .
$$

Let $S_{1}:=[n] \backslash\left\{t_{1}\right\}$. Then

$$
\begin{aligned}
I\left(X_{1}^{n} ; X_{2}^{n} \mid U\right) \\
\quad=I\left(X_{1}^{n} ; X_{2 t_{1}} \mid U\right)+I\left(X_{1}^{n} ; X_{2}^{S_{1}} \mid U, X_{2 t_{1}}\right) \\
=I\left(X_{1 t_{1}} ; X_{2 t_{1}} \mid U\right)+I\left(X_{1}^{S_{1}} ; X_{2 t_{1}} \mid U, X_{1 t_{1}}\right) \\
\quad+I\left(X_{1 t_{1}} ; X_{2}^{S_{1}} \mid U, X_{2 t_{1}}\right)+I\left(X_{1}^{S_{1}} ; X_{2}^{S_{1}} \mid U, X_{1 t_{1}}, X_{2 t_{1}}\right) \\
\quad \geq I\left(X_{1 t_{1}} ; X_{2 t_{1}} \mid U\right)+I\left(X_{1}^{S_{1}} ; X_{2}^{S_{1}} \mid U, X_{1 t_{1}}, X_{2 t_{1}}\right) .
\end{aligned}
$$

Since $I\left(X_{1}^{n} ; X_{2}^{n} \mid U\right) \leq n \delta$, applying 42 gives

$$
I\left(X_{1}^{S_{1}} ; X_{2}^{S_{1}} \mid U, X_{1 t_{1}}, X_{2 t_{1}}\right) \leq n \delta-\epsilon .
$$

Now if for all $t \in S_{1}$,

$$
I\left(X_{1 t} ; X_{2 t} \mid U, X_{1 t_{1}}, X_{2 t_{1}}\right) \leq \epsilon,
$$

then we define $T:=\left\{t_{1}\right\}$. Otherwise, there exists $t_{2} \in[n]$ such that

$$
I\left(X_{1 t_{2}} ; X_{2 t_{2}} \mid U, X_{1 t_{1}}, X_{2 t_{1}}\right)>\epsilon .
$$

Similar to the above argument, if we define $S_{2}:=[n] \backslash\left\{t_{1}, t_{2}\right\}$, then

$$
I\left(X_{1}^{S_{2}} ; X_{2}^{S_{2}} \mid U, X_{1 t_{1}}, X_{1 t_{2}}, X_{2 t_{1}}, X_{2 t_{2}}\right) \leq n \delta-2 \epsilon .
$$

If we continue this process, we eventually get a set $T:=$ $\left\{t_{1}, \ldots, t_{k}\right\}$ such that

$$
I\left(X_{1}^{T^{c}} ; X_{2}^{T^{c}} \mid U, X_{1}^{T}, X_{2}^{T}\right) \leq n \delta-|T| \epsilon,
$$

and for all $t \in S_{k}:=T^{c}$,

$$
I\left(X_{1 t} ; X_{2 t} \mid U, X_{1}^{T}, X_{2}^{T}\right) \leq \epsilon .
$$

In addition, from (43) it follows that

$$
|T| \leq \frac{n \delta}{\epsilon} .
$$

\section{H. Proof of Corollary 14}

Here we give an upper bound for $\sigma(\delta)$, which is defined by 9), in terms of $\sigma_{1}(\delta)$.

Fix a positive integer $n$. By Lemma 15, we can choose $\mathcal{U}=\{a, b\}$ in the definition of $\sigma_{n}(\delta)$. From Lemma 13 , it follows that there exists a set $T \subseteq[n]$ such that

$$
0 \leq|T| \leq \frac{n \delta}{\epsilon},
$$

and

$$
\forall t \notin T: I\left(X_{1 t} ; X_{2 t} \mid U, X_{1}^{T}, X_{2}^{T}\right) \leq \epsilon .
$$

Thus

$$
\begin{aligned}
& I\left(X_{1}^{n}, X_{2}^{n} ; Y^{n} \mid U\right) \\
& =I\left(X_{1}^{T}, X_{2}^{T} ; Y^{n} \mid U\right)+I\left(X_{1}^{T^{c}}, X_{2}^{T^{c}} ; Y^{n} \mid U, X_{1}^{T}, X_{2}^{T}\right) \\
& \leq|T| \log \left|\mathcal{X}_{1}\right|\left|\mathcal{X}_{2}\right|+I\left(X_{1}^{T^{c}}, X_{2}^{T^{c}} ; Y^{n} \mid U, X_{1}^{T}, X_{2}^{T}\right) .
\end{aligned}
$$

Next define

$$
\mathcal{V}:=\mathcal{U} \times \mathcal{X}_{1}^{|T|} \times \mathcal{X}_{2}^{|T|}
$$


We further bound the second term on the right hand side of (46) by

$$
\begin{aligned}
I\left(X_{1}^{T^{c}}, X_{2}^{T^{c}} ; Y^{n} \mid U, X_{1}^{T}, X_{2}^{T}\right) & \sum_{t \in[n]} I\left(X_{1}^{T^{c}}, X_{2}^{T^{c}} ; Y_{t} \mid U, X_{1}^{T}, X_{2}^{T}, Y^{t-1}\right) \\
= & \sum_{t \in[n]}\left[H\left(Y_{t} \mid U, X_{1}^{T}, X_{2}^{T}, Y^{t-1}\right)\right. \\
& \left.-H\left(Y_{t} \mid U, X_{1}^{n}, X_{2}^{n}, Y^{t-1}\right)\right] \\
\leq & \sum_{t \in[n]}\left[H\left(Y_{t} \mid U, X_{1}^{T}, X_{2}^{T}\right)\right. \\
& \left.-H\left(Y_{t} \mid U, X_{1}^{n}, X_{2}^{n}, Y^{t-1}\right)\right] \\
= & \sum_{t \in[n]}\left[H\left(Y_{t} \mid U, X_{1}^{T}, X_{2}^{T}\right)\right. \\
& \left.-H\left(Y_{t} \mid U, X_{1}^{T}, X_{2}^{T}, X_{1 t}, X_{2 t}\right)\right] \\
= & \sum_{t \in[n]} I\left(X_{1 t}, X_{2 t} ; Y_{t} \mid U, X_{1}^{T}, X_{2}^{T}\right) \\
\leq & \max _{p \in \mathcal{P}_{\mathcal{V}}^{(1)}(\epsilon)} I\left(X_{1}, X_{2} ; Y \mid V\right) \leq n \sigma_{1}(\epsilon),
\end{aligned}
$$

where 47) follows from the chain rule for mutual information, (48) follows from the fact that conditioning reduces entropy, 49. follows from the fact that for every $\left(u, x_{1}^{n}, x_{2}^{n}, y^{t}\right)$,

$$
\begin{aligned}
p\left(y_{t} \mid u, x_{1}^{n}, x_{2}^{n}, y^{t-1}\right) & =\frac{p\left(u, x_{1}^{n}, x_{2}^{n}, y^{t}\right)}{p\left(u, x_{1}^{n}, x_{2}^{n}, y^{t-1}\right)} \\
& =\frac{p\left(y^{t} \mid u, x_{1}^{n}, x_{2}^{n}\right)}{p\left(y^{t-1} \mid u, x_{1}^{n}, x_{2}^{n}\right)} \\
& =\frac{p\left(y^{t} \mid x_{1}^{t}, x_{2}^{t}\right)}{p\left(y^{t-1} \mid x_{1}^{t-1}, x_{2}^{t-1}\right)} \\
& =p\left(y_{t} \mid x_{1 t}, x_{2 t}\right),
\end{aligned}
$$

and similarly,

$$
p\left(y_{t} \mid u, x_{1}^{T}, x_{2}^{T}, x_{1 t}, x_{2 t}\right)=p\left(y_{t} \mid x_{1 t}, x_{2 t}\right) .
$$

Therefore, by (45), 46, and (50),

$$
\frac{1}{n} I\left(X_{1}^{n}, X_{2}^{n} ; Y^{n} \mid U\right) \leq \frac{\delta}{\epsilon} \log \left|\mathcal{X}_{1}\right|\left|\mathcal{X}_{2}\right|+\sigma_{1}(\epsilon),
$$

which completes the proof.

\section{Proof of Lemma 15}

To simplify notation, we prove this lemma for the case $n=$ 1. The same proof works for any $n>1$.

Here we show that in the definition of $\sigma_{1}$,

$$
\sigma_{1}(\delta):=\sup _{\mathcal{U}} \max _{p \in \mathcal{P}_{\mathcal{U}}^{(n)}(\delta)} I\left(X_{1}, X_{2} ; Y \mid U\right)
$$

where the supremum is over all finite sets $\mathcal{U}$, we can remove the supremum and limit $\mathcal{U}$ to have cardinality at most two.
Let $\mathcal{U}$ be some finite set and let $p^{*}\left(u, x_{1}, x_{2}\right) \in \mathcal{P}_{\mathcal{U}}^{(1)}(\delta)$ be a distribution that satisfies

$$
I^{*}\left(X_{1}, X_{2} ; Y \mid U\right)=\max _{p \in \mathcal{P}_{\mathcal{U}}^{(1)}(\delta)} I\left(X_{1}, X_{2} ; Y \mid U\right),
$$

where in 51 and below, mutual information terms with the superscript ' $*$ ' are calculated with respect to $p^{*}\left(u, x_{1}, x_{2}\right) p\left(y \mid x_{1}, x_{2}\right)$ and its marginals. Let $\mathcal{Q} \subseteq \mathbb{R}^{|\mathcal{U}|}$ denote the set of all vectors $(q(u))_{u \in \mathcal{U}}$ that satisfy

$$
\begin{aligned}
& q(u) \geq 0 \text { for all } u \in \mathcal{U} \\
& \sum_{u \in \mathcal{U}} q(u)=1 \\
& \sum_{u \in \mathcal{U}} q(u) I^{*}\left(X_{1} ; X_{2} \mid U=u\right)=I^{*}\left(X_{1} ; X_{2} \mid U\right) .
\end{aligned}
$$

Note that $\mathcal{Q}$ is nonempty since $p^{*}(u) \in \mathcal{Q}$. Consider the mapping $F: \mathcal{Q} \rightarrow \mathbb{R}_{\geq 0}$ defined by

$$
F[q]:=\sum_{u \in \mathcal{U}} q(u) I^{*}\left(X_{1}, X_{2} ; Y \mid U=u\right) .
$$

Since $p^{*}(u) \in \mathcal{Q}$ and for all $q(u) \in \mathcal{Q}$, by 52 we have $q(u) p^{*}\left(x_{1}, x_{2} \mid u\right) \in \mathcal{P}_{\mathcal{U}}^{(1)}(\delta)$, thus

$$
\max _{q \in \mathcal{Q}} F[q]=\max _{p \in \mathcal{P}_{\mathcal{U}}^{(1)}(\delta)} I\left(X_{1}, X_{2} ; Y \mid U\right) .
$$

Therefore, it suffices to find $q^{*} \in \mathcal{Q}$ which has at most two non-zero components and at which $F$ obtains its maximal value. To this end, note that since $\mathcal{Q}$ is a nonempty bounded polyhedron, by [28, p. 65, Corollary 2.2] and [28, p. 50, Theorem 2.3], $\mathcal{Q}$ has at least one extreme point. Since $F$ is linear in $q$ and $\mathcal{Q}$ has at least one extreme point, [28, p. 65, Theorem 2.7] shows that there exists an extreme point of $\mathcal{Q}$, say $q^{*} \in \mathcal{Q}$, at which $F$ obtains its maximum. Finally, since $q^{*}$ is an extreme point, applying [28, p. 50, Theorem 2.3] to the definition of $\mathcal{Q}$ implies that we must have $q^{*}(u)=0$ for at least $|\mathcal{U}|-2$ values of $u$. This completes the proof.

\section{J. Proof of Lemma 16}

We next show that $\sigma_{1}(\delta)$ is continuous at $\delta=0$. By Lemma 15. without loss of generality, we can remove the supremum over $\mathcal{U}$ and set $\mathcal{U}$ to be a fixed, finite set. Then for all $\delta \geq 0$, we have

$$
\sigma_{1}(\delta)=\max _{p \in \mathcal{P}_{\mathcal{U}}^{(1)}(\delta)} I\left(X_{1}, X_{2} ; Y \mid U\right)
$$

Fix $\delta>0$. Let $p^{*}\left(u, x_{1}, x_{2}\right)$ be a distribution in $\mathcal{P}_{\mathcal{U}}^{(1)}(\delta)$ achieving the maximum above, and define

$$
p_{\text {ind }}^{*}\left(x_{1}, x_{2} \mid u\right):=p^{*}\left(x_{1} \mid u\right) p^{*}\left(x_{2} \mid u\right) .
$$

In addition, for each $u \in \mathcal{U}$, let

$$
\begin{gathered}
D\left(p^{*}\left(x_{1}, x_{2} \mid u\right) \| p_{\text {ind }}^{*}\left(x_{1}, x_{2} \mid u\right)\right) \\
=\sum_{x_{1}, x_{2}} p^{*}\left(x_{1}, x_{2} \mid u\right) \log \frac{p^{*}\left(x_{1}, x_{2} \mid u\right)}{p_{\text {ind }}^{*}\left(x_{1}, x_{2} \mid u\right)}, \\
\left\|p^{*}(y \mid u)-p_{\text {ind }}^{*}(y \mid u)\right\|_{L^{1}}=\sum_{y}\left|p^{*}(y \mid u)-p_{\text {ind }}^{*}(y \mid u)\right|,
\end{gathered}
$$


and

$$
\begin{aligned}
& \left\|p^{*}\left(x_{1}, x_{2} \mid u\right)-p_{\text {ind }}^{*}\left(x_{1}, x_{2} \mid u\right)\right\|_{L^{1}} \\
& \quad=\sum_{x_{1}, x_{2}}\left|p^{*}\left(x_{1}, x_{2} \mid u\right)-p_{\text {ind }}^{*}\left(x_{1}, x_{2} \mid u\right)\right| .
\end{aligned}
$$

Since

$$
\begin{aligned}
& \sum_{u \in \mathcal{U}} p^{*}(u) D\left(p^{*}\left(x_{1}, x_{2} \mid u\right) \| p_{\text {ind }}^{*}\left(x_{1}, x_{2} \mid u\right)\right) \\
& =I^{*}\left(X_{1} ; X_{2} \mid U\right) \leq \delta
\end{aligned}
$$

by applying [3, Lemma 11.6.1] for every $u \in \mathcal{U}$, we get

$$
\sum_{u \in \mathcal{U}} p^{*}(u)\left\|p^{*}\left(x_{1}, x_{2} \mid u\right)-p_{\text {ind }}^{*}\left(x_{1}, x_{2} \mid u\right)\right\|_{L^{1}}^{2} \leq 2 \delta \ln 2 .
$$

In addition,

$$
\begin{aligned}
& \sum_{u \in \mathcal{U}} p^{*}(u)\left\|p^{*}(y \mid u)-p_{\text {ind }}^{*}(y \mid u)\right\|_{L^{1}} \\
& \leq \sum_{u \in \mathcal{U}} p^{*}(u) \sum_{x_{1}, x_{2}} p\left(y \mid x_{1}, x_{2}\right)\left|p^{*}\left(x_{1}, x_{2} \mid u\right)-p_{\text {ind }}^{*}\left(x_{1}, x_{2} \mid u\right)\right| \\
& \leq \sum_{u \in \mathcal{U}} p^{*}(u)\left\|p^{*}\left(x_{1}, x_{2} \mid u\right)-p_{\text {ind }}^{*}\left(x_{1}, x_{2} \mid u\right)\right\|_{L^{1}} \\
& \leq \sqrt{2 \delta \ln 2}
\end{aligned}
$$

where (55) follows from (54) and the Cauchy-Schwarz inequality. Define the subset $\mathcal{U}_{0} \subseteq \mathcal{U}$ as

$$
\mathcal{U}_{0}=\left\{u \in \mathcal{U}:\left\|p^{*}(y \mid u)-p_{\text {ind }}^{*}(y \mid u)\right\|_{L^{1}} \leq 1 / 2\right\} .
$$

Clearly, by [55,

$$
\sum_{u \notin \mathcal{U}_{0}} p^{*}(u) \leq 2 \sqrt{2 \delta \ln 2} .
$$

Thus

$$
\begin{aligned}
& \left|H^{*}(Y \mid U)-H_{\text {ind }}^{*}(Y \mid U)\right| \\
& \leq \sum_{u \in \mathcal{U}} p^{*}(u)\left|H^{*}(Y \mid U=u)-H_{\text {ind }}^{*}(Y \mid U=u)\right| \\
& \leq 2 \sqrt{2 \delta \ln 2} \log |\mathcal{Y}| \\
& \quad-\sum_{u \in \mathcal{U}_{0}}\left[p^{*}(u)\left\|p^{*}(y \mid u)-p_{\text {ind }}^{*}(y \mid u)\right\|_{L^{1}}\right. \\
& \left.\quad \times \log \frac{\left\|p^{*}(y \mid u)-p_{\text {ind }}^{*}(y \mid u)\right\|_{L^{1}}}{|\mathcal{Y}|}\right] \\
& \leq 2 \sqrt{2 \delta \ln 2} \log |\mathcal{Y}| \\
& -\sum_{u \in \mathcal{U}}\left[p^{*}(u)\left\|p^{*}(y \mid u)-p_{\text {ind }}^{*}(y \mid u)\right\|_{L^{1}}\right. \\
& \quad \times \log \frac{\left\|p^{*}(y \mid u)-p_{\text {ind }}^{*}(y \mid u)\right\|_{L^{1}}}{|\mathcal{Y}|} \\
& \leq 2 \sqrt{2 \delta \ln 2} \log |\mathcal{Y}|-\sqrt{2 \delta \ln 2} \log \left(\frac{1}{|\mathcal{Y}|} \sqrt{2 \delta \ln 2}\right) \\
& =\sqrt{2 \delta \ln 2} \log \frac{|\mathcal{Y}|^{3}}{\sqrt{2 \delta \ln 2}},
\end{aligned}
$$

where (57) follows from (56) and [3, Theorem 17.3.3], (58) follows from the fact that for all $u \in \mathcal{U}$,

$-\left\|p^{*}(y \mid u)-p_{\text {ind }}^{*}(y \mid u)\right\|_{L^{1}} \log \frac{\left\|p^{*}(y \mid u)-p_{\text {ind }}^{*}(y \mid u)\right\|_{L^{1}}}{|\mathcal{Y}|} \geq 0$, and for $\delta$ satisfying

$$
0<\sqrt{2 \delta \ln 2}<\frac{1}{e}|\mathcal{Y}|,
$$

59 follows from 55 and the fact that the mapping $t \mapsto-t \log (t /|\mathcal{Y}|)$ is concave on $(0, \infty)$ and increasing on $(0,|\mathcal{Y}| / e)$. In addition, by (55),

$$
\begin{aligned}
& \left|H^{*}\left(Y \mid U, X_{1}, X_{2}\right)-H_{\text {ind }}^{*}\left(Y \mid U, X_{1}, X_{2}\right)\right| \\
& \leq \sum_{u, x_{1}, x_{2}}\left[\left|p^{*}\left(u, x_{1}, x_{2}\right)-p_{\text {ind }}^{*}\left(u, x_{1}, x_{2}\right)\right|\right. \\
& \left.\quad \times H\left(Y \mid X_{1}=x_{1}, X_{2}=x_{2}\right)\right] \\
& \leq \sum_{u, x_{1}, x_{2}}\left|p^{*}\left(u, x_{1}, x_{2}\right)-p_{\text {ind }}^{*}\left(u, x_{1}, x_{2}\right)\right| \log |\mathcal{Y}| \\
& =(\log |\mathcal{Y}|) \cdot \sum_{u \in \mathcal{U}} p^{*}(u)\left\|p^{*}\left(x_{1}, x_{2} \mid u\right)-p_{\text {ind }}^{*}\left(x_{1}, x_{2} \mid u\right)\right\|_{L^{1}} \\
& \leq(\log |\mathcal{Y}|) \sqrt{2 \delta \ln 2} .
\end{aligned}
$$

Thus by (60) and (62), for all $\delta$ satisfying (61),

$$
\begin{aligned}
\sigma_{1}(\delta)= & I^{*}\left(X_{1}, X_{2} ; Y \mid U\right)=H^{*}(Y \mid U)-H^{*}\left(Y \mid U, X_{1}, X_{2}\right) \\
\leq & \left|H^{*}(Y \mid U)-H_{\text {ind }}^{*}(Y \mid U)\right| \\
& +\left|H^{*}\left(Y \mid U, X_{1}, X_{2}\right)-H_{\text {ind }}^{*}\left(Y \mid U, X_{1}, X_{2}\right)\right| \\
& +I_{\text {ind }}^{*}\left(X_{1}, X_{2} ; Y \mid U\right) \\
\leq & \sqrt{2 \delta \ln 2} \log \frac{|\mathcal{Y}|^{3}}{\sqrt{2 \delta \ln 2}}+(\log |\mathcal{Y}|) \sqrt{2 \delta \ln 2}+\sigma_{1}(0) .
\end{aligned}
$$

Since $\sigma_{1}(0) \leq \sigma_{1}(\delta)$ for all $\delta \geq 0$, the continuity of $\sigma_{1}$ at $\delta=0^{+}$follows.

\section{K. Proof of Claim 19}

The result follows from the definition of $W^{n_{1}}$. Consider entry $i$ of $w_{2} \in\{0,1\}^{n_{1}}, w_{2, i} \in\{0,1\}$, and its corresponding entry $\bar{w}_{2, i} \in\{0,1\}$ in $\bar{w}_{2} \in\{0,1\}^{n_{1}}$. Let $x_{1}^{n_{1}}=f_{1,1}\left(w_{1}\right)$, and let $x_{1, i}$ be entry $i$ of $x_{1}^{n_{1}}$. It holds (by a simple exhaustive case analysis) that exactly one of the values $W_{1}\left(x_{1, i}, w_{2, i}\right)$ and $W_{1}\left(x_{1, i}, \bar{w}_{2, i}\right)$ is in the set $\{c, C\}$. Thus, the number of entries in $W_{1}^{n_{1}}\left(f_{1,1}\left(w_{1}\right), w_{2}\right)$ that are in the set $\{c, C\}$ plus the number of entries in $W_{1}^{n_{1}}\left(f_{1,1}\left(w_{1}\right), \bar{w}_{2}\right)$ that are in the set $\{c, C\}$ equals $n_{1}$. If the former exceeds $n_{1} / 2$ (i.e., $W^{n_{1}}\left(f_{1,1}\left(w_{1}\right), w_{2}\right)$ is bad $)$ then the latter is less than $n_{1} / 2$ (i.e., $W^{n_{1}}\left(f_{1,1}\left(w_{1}\right), \bar{w}_{2}\right)$ is good).

\section{Proof of Claim 20}

Consider a good pair $\left(y_{1}^{n_{1}}, y_{2}^{n_{1}}\right)$. As the pair $\left(y_{1}^{n_{1}}, y_{2}^{n_{1}}\right)$ is good, we start by noting that the set $\left(W^{-1}\right)^{n_{1}}\left(y_{1}^{n_{1}}, y_{2}^{n_{1}}\right)$ of preimages $\left(x_{1}^{n_{1}}, x_{2}^{n_{1}}\right) \in \mathcal{X}_{1}^{n} \times \mathcal{X}_{2}^{n}$ for which $W^{n_{1}}\left(x_{1}^{n_{1}}, x_{2}^{n_{1}}\right)=$ $\left(y_{1}^{n_{1}}, y_{2}^{n_{1}}\right)$ satisfies that $x_{2}^{n_{1}}=y_{2}^{n_{1}}$ and that $x_{1}^{n_{1}}$ must be one of at most $2^{n_{1} / 2}$ vectors in $\mathcal{X}_{1}^{n_{1}}$. Denote this latter subset of $\mathcal{X}_{1}^{n_{1}}$ by $\operatorname{Pre}_{1}\left(y_{1}^{n_{1}}\right)$. Thus, the number of messages $w_{1}$ such 
that $W^{n_{1}}\left(f_{1,1}\left(w_{1}\right), y_{2}^{n_{1}}\right)=\left(y_{1}^{n_{1}}, y_{2}^{n_{1}}\right)$ equals the number of messages $w_{1}$ for which $f_{1,1}\left(w_{1}\right) \in \operatorname{Pre}_{1}\left(y_{1}^{n_{1}}\right)$. We analyze this latter quantity.

Recall that the codebook $\left\{x_{1}^{n_{1}}\left(w_{1}\right)\right\}_{w_{1} \in\left[2^{n R_{1}}\right]}$ is chosen uniformly at random from the collection of all subsets of size $2^{n R_{1}}$ of $\mathcal{X}_{1}^{n_{1}}$. Namely, one can choose the codebook $\left\{x_{1}^{n_{1}}\left(w_{1}\right)\right\}_{w_{1} \in\left[2^{n R_{1}}\right]}$ in an iterative manner, where in iteration $w_{1}, x_{1}^{n_{1}}\left(w_{1}\right)$ is chosen uniformly from $\mathcal{X}_{1}^{n_{1}} \backslash$ $\left\{x_{1}^{n_{1}}\left(w_{1}^{\prime}\right)\right\}_{w_{1}^{\prime}<w_{1}}$. Here, we use the standard order on $\left[2^{n R_{1}}\right]$. For any $w_{1}$, conditioning on the value of $f_{1,1}\left(w_{1}^{\prime}\right)$ for all $w_{1}^{\prime}<$ $w_{1}$, the probability (over the choice of $f_{1,1}\left(w_{1}\right)=x_{1}^{n_{1}}\left(w_{1}\right)$ ) that $f_{1,1}\left(w_{1}\right) \in \operatorname{Pre}_{1}\left(y_{1}^{n_{1}}\right)$ is at most

$$
\frac{2^{n_{1} / 2}}{4^{n_{1}}-2^{3 n_{1} / 2-\delta n_{1}}} \leq 2 \cdot 2^{-3 n_{1} / 2}
$$

for sufficiently large $n$. We use the observations that $n_{1}=$ $(1-\epsilon) n$, that in each iteration at most $2^{n R_{1}}=2^{n_{1}(3 / 2-\delta)}$ codewords have been chosen so far, and that our choices are without repetition. Thus, the probability that there exist $\ell$ messages $\left\{w_{1,1}, \ldots, w_{1, \ell}\right\}$ in $\left[2^{n R_{1}}\right]$ such that for all $i=$ $1, \ldots, \ell$ it holds that $f_{1,1}\left(w_{1, i}\right) \in \operatorname{Pre}_{1}\left(y_{1}^{n_{1}}\right)$ is at most

$$
\begin{aligned}
\left(\begin{array}{c}
2^{3 n_{1} / 2-\delta n_{1}} \\
\ell
\end{array}\right) 2^{\ell} 2^{-3 \ell n_{1} / 2} & \leq 2^{3 \ell n_{1} / 2-\delta n_{1} \ell} 2^{\ell} 2^{-3 \ell n_{1} / 2} \\
& =2^{\ell} 2^{-\delta n_{1} \ell}
\end{aligned}
$$

Setting $\ell=\lceil 3 / \delta\rceil+1$ and allowing $n$ (and thus $n_{1}$ ) to grow sufficiently large, we find that the above probability is at most $2^{-3 n_{1}}$. Finally, taking the union bound over possible $y_{1}^{n_{1}}$, we conclude that with probability at least $1-\left|\mathcal{Y}_{1}\right|^{n_{1}} \cdot 2^{-3 n_{1}}=$ $1-2^{-n_{1}(3-\log 6)} \geq 1-2^{-0.4 n_{1}}$ (over $f_{1: 1}$ ) for any good pair $\left(y_{1}^{n_{1}}, y_{2}^{n_{1}}\right)$, the number of messages $w_{1}$ for which $f_{1,1}\left(w_{1}\right) \in$ $\operatorname{Pre}_{1}\left(y_{1}^{n_{1}}\right)$ is bounded by $\lceil 3 / \delta\rceil$.

\section{Proof of Lemma 21}

Here we show that for each fixed $\mathbf{C}_{\text {out }} \in \mathbb{R}_{\geq 0}^{2}$, $C_{\text {sum }}\left(\mathbf{C}_{\text {in }}, \mathbf{C}_{\text {out }}\right)$ is continuous with respect to $\mathbf{C}_{\mathrm{in}}$. To this end, fix $\mathbf{C}_{\text {out }} \in \mathbb{R}_{\geq 0}^{2}$ and define the functions $f, g: \mathbb{R}_{\geq 0}^{2} \rightarrow \mathbb{R}$ as

$$
\begin{aligned}
f\left(\mathbf{C}_{\text {in }}\right) & :=C_{\text {sum }}\left(\mathbf{C}_{\text {in }}, \mathbf{C}_{\text {out }}\right)-C_{\text {sum }}\left(\mathbf{C}_{\text {in }}, \mathbf{0}\right) \\
& =C_{\text {sum }}\left(\mathbf{C}_{\text {in }}, \mathbf{C}_{\text {out }}\right)-C_{\text {sum }}(\mathbf{0}, \mathbf{0})
\end{aligned}
$$

and

$$
g\left(\mathbf{C}_{\text {in }}\right):=C_{\text {in }}^{1}+C_{\text {in }}^{2}-f\left(\mathbf{C}_{\text {in }}\right) .
$$

By a time-sharing argument [23. Lemma 4], it follows that $f$ is concave, and consequently, $g$ is convex. Thus for all $\lambda \in[0,1]$ and all $\left(\mathbf{C}_{\text {in }}, \tilde{\mathbf{C}}_{\text {in }}\right)$,

$$
g\left(\lambda \mathbf{C}_{\text {in }}+(1-\lambda) \tilde{\mathbf{C}}_{\text {in }}\right) \leq \lambda g\left(\mathbf{C}_{\text {in }}\right)+(1-\lambda) g\left(\tilde{\mathbf{C}}_{\text {in }}\right) .
$$

Since $g(\mathbf{0})=0$, setting $\tilde{\mathbf{C}}_{\text {in }}=\mathbf{0}$ gives

$$
g\left(\lambda \mathbf{C}_{\text {in }}\right) \leq \lambda g\left(\mathbf{C}_{\text {in }}\right)
$$

Note that by [6, Proposition 6], $g$ is nonnegative. Thus

$$
g\left(\lambda \mathbf{C}_{\text {in }}\right) \leq g\left(\mathbf{C}_{\text {in }}\right),
$$

which, when written in terms of $f$, becomes

$$
f\left(\mathbf{C}_{\mathrm{in}}\right)-f\left(\lambda \mathbf{C}_{\mathrm{in}}\right) \leq(1-\lambda)\left(C_{\mathrm{in}}^{1}+C_{\mathrm{in}}^{2}\right) .
$$

Consider $\mathbf{C}_{\text {in }}, \tilde{\mathbf{C}}_{\text {in }} \in \mathbb{R}_{\geq 0}^{2}$. Define the pairs $\mathbf{C}_{\mathrm{in}, \min }, \mathbf{C}_{\mathrm{in}, \max } \in \mathbb{R}_{\geq 0}^{2}$ as

$$
\begin{aligned}
& \forall i \in\{1,2\}: C_{\mathrm{in}, \min }^{i}:=\min \left\{C_{\mathrm{in}}^{i}, \tilde{C}_{\mathrm{in}}^{i}\right\} \\
& \forall i \in\{1,2\}: C_{\mathrm{in}, \max }^{i}:=\max \left\{C_{\mathrm{in}}^{i}, \tilde{C}_{\mathrm{in}}^{i}\right\} .
\end{aligned}
$$

Next define $\lambda^{*}\left(\mathbf{C}_{\text {in }}, \tilde{\mathbf{C}}_{\text {in }}\right) \in[0,1]$ as 6

$$
\lambda^{*}:=\min _{i \in\{1,2\}} C_{\mathrm{in}, \min }^{i} / C_{\mathrm{in}, \max }^{i} .
$$

Then

$$
\begin{aligned}
& \left|f\left(\mathbf{C}_{\text {in }}\right)-f\left(\tilde{\mathbf{C}}_{\text {in }}\right)\right| \\
& \leq\left|f\left(\mathbf{C}_{\text {in }}\right)-f\left(\mathbf{C}_{\text {in }, \text { min }}\right)\right|+\left|f\left(\mathbf{C}_{\text {in }, \text { min }}\right)-f\left(\tilde{\mathbf{C}}_{\text {in }}\right)\right| \\
& =f\left(\mathbf{C}_{\text {in }}\right)-f\left(\mathbf{C}_{\text {in }, \text { min }}\right)+f\left(\tilde{\mathbf{C}}_{\text {in }}\right)-f\left(\mathbf{C}_{\text {in }, \text { min }}\right) \\
& \leq f\left(\mathbf{C}_{\text {in }}\right)-f\left(\lambda^{*} \mathbf{C}_{\text {in }}\right)+f\left(\tilde{\mathbf{C}}_{\text {in }}\right)-f\left(\lambda^{*} \tilde{\mathbf{C}}_{\text {in }}\right) \\
& \leq\left(1-\lambda^{*}\right)\left(C_{\text {in }}^{1}+C_{\text {in }}^{2}\right)+\left(1-\lambda^{*}\right)\left(\tilde{C}_{\text {in }}^{1}+\tilde{C}_{\text {in }}^{2}\right),
\end{aligned}
$$

where (64) follows from the triangle inequality, 650 follows from the definition of $\mathbf{C}_{\mathrm{in}, \mathrm{min}},(66)$ follows from the definition of $\lambda^{*}$, and 67) follows from 63). Finally, if we let $\tilde{\mathbf{C}}_{\mathrm{in}} \rightarrow \mathbf{C}_{\mathrm{in}}$ in 67, we see that $f\left(\tilde{\mathbf{C}}_{\text {in }}\right) \rightarrow f\left(\mathbf{C}_{\text {in }}\right)$ since

$$
\lim _{\tilde{\mathbf{C}}_{\mathrm{in}} \rightarrow \mathbf{C}_{\mathrm{in}}} \lambda^{*}\left(\mathbf{C}_{\mathrm{in}}, \tilde{\mathbf{C}}_{\mathrm{in}}\right)=1 \text {. }
$$

\section{N. Proof of Lemma 24}

First note that due to the symmetry between $x$ and $y$, we may, without the loss of generality, assume that $0 \leq x \leq y$. Then under the lemma assumption, we have

$$
0 \leq y-x \leq x \leq y \text {. }
$$

We further remark that in the cases where equality holds in any of the inequalities of 68), the result holds trivially. Thus we only need to consider the case where all of the inequalities in 68 are strict, giving

$$
0<y-x<x<y
$$

From here, the result follows directly by applying a wellknown property of concave functions: if $g:[a, b] \rightarrow \mathbb{R}$ is a concave function, then for any $r, s, t \in[a, b]$ satisfying $r<$ $s<t$, we have

$$
\frac{g(t)-g(s)}{t-s} \leq \frac{g(s)-g(r)}{s-r} .
$$

Note that 69 follows directly from applying the definition of a concave function to the convex combination

$$
s=\frac{t-s}{t-r} \cdot r+\frac{s-r}{t-r} \cdot t
$$

Using 696 twice, we can derive our desired result. First by setting $r=0, s=y-x$, and $t=x$, we get

$$
\frac{f(x)-f(y-x)}{x-(y-x)} \leq \frac{f(y-x)-f(0)}{y-x} .
$$

${ }^{6}$ If for some $i \in\{1,2\}$, say $i=1, C_{\mathrm{in}, \max }^{i}=0$, set $\lambda^{*}:=$ $\min \left\{1, C_{\mathrm{in}, \min }^{2} / C_{\mathrm{in}, \max }^{2}\right\}$. If $C_{\mathrm{in}, \max }^{1}=C_{\mathrm{in}, \max }^{2}=0$, set $\lambda^{*}=1$. These definitions ensure the continuity of $\lambda^{*}$ in $\left(\mathbf{C}_{\mathrm{in}}, \tilde{\mathbf{C}}_{\mathrm{in}}\right)$. 
Applying (69) for the second time, this time by setting $r=$ $y-x, s=x$, and $t=y$, we get

$$
\frac{f(y)-f(x)}{y-x} \leq \frac{f(x)-f(y-x)}{x-(y-x)}
$$

Finally, combining (70) with (71) and multiplying both sides by $y-x$ gives the desired result.

\section{Summary}

Consider a network consisting of a discrete MAC and a $\mathrm{CF}$ that has full knowledge of the messages. In this work, we show that the average-error sum-capacity of such a network is always a continuous function of the CF output link capacities; this is in contrast to our previous results on the continuity of maximal-error sum-capacity [5] and our current result using only a constant number of cooperation bits. For the average case analysis, our proof method relies on finding lower and upper bounds on the sum-capacity and then using a modified version of a technique developed by Dueck [19] to demonstrate continuity.

Our result on maximal-error considers first the maximalerror list-decoding capacity, and then reduces list-decoding to unique-decoding. Our result strongly relies on the precise functionality of Dueck's MAC [17] and on the fact that it has a maximal-error capacity region that differs from its averageerror capacity region. A deeper understanding of the family of MACs for which a constant number of bits (or even a single bit) of cooperation can affect the maximal-error capacity region is left for future research.

In the general case where the CF may only have partial knowledge of messages, we 1) characterize the growth rate of the average-error sum-capacity as a function of the CF output link capacities, 2) demonstrate that the average-error sumcapacity continuity problem can be significantly simplified, and 3) we characterize the set of all discrete MACs where the maximal-error sum-capacity is not continuous in the $\mathrm{CF}$ output link capacities.

Our study of the MAC with a CF can also be formulated as an edge removal problem in general multi-terminal networks. The latter problem opens the door to a host of related questions. Each question seeks to determine whether adding a $\delta$-capacity noiseless channel to a memoryless network results in an average-error capacity region that is not continuous at $\delta=0$. These questions help us pinpoint whether the addition of asymptotically negligible cooperation can ever have a non-negligible impact on average-error capacity as it can on maximal-error capacity.

\section{APPENDIX}

Our goal here is to show that in the definition of $\mathcal{C}^{*}$ from Section [V] the condition

$$
I_{\mathrm{dep}}\left(X_{1}, X_{2} ; Y\right)+D\left(p_{\mathrm{dep}}(y) \| p_{\text {ind }}(y)\right)>I_{\text {ind }}\left(X_{1}, X_{2} ; Y\right) .
$$

can be replaced with

$$
I_{\text {dep }}\left(X_{1}, X_{2} ; Y\right)>I_{\text {ind }}\left(X_{1}, X_{2} ; Y\right) .
$$

To this end, let $\mathcal{C}$ be defined analogously to $\mathcal{C}^{*}$ with 73 replacing (72). First note that since (73) implies (72), we have $\mathcal{C} \subseteq \mathcal{C}^{*}$. Thus it suffices to show that $\mathcal{C}^{*} \subseteq \mathcal{C}$.

Let $\left(\mathcal{X}_{1} \times \mathcal{X}_{2}, p\left(y \mid x_{1}, x_{2}\right), \mathcal{Y}\right)$ be a MAC not in $\mathcal{C}$; we show that it is also not in $\mathcal{C}^{*}$. Since this MAC is not in $\mathcal{C}$,

$$
\max _{p_{\text {dep }}\left(x_{1}, x_{2}\right)} I_{\text {dep }}\left(X_{1}, X_{2} ; Y\right)=I_{\text {ind }}\left(X_{1}, X_{2} ; Y\right),
$$

where in (74), the maximum is over all distributions $p_{\text {dep }}$ whose support is contained in the support of $p_{\text {ind }}$. Denote the latter set as $\mathcal{S}_{\text {ind }}$. Then for the point-to-point channel $\left(\mathcal{S}_{\text {ind }}, p\left(y \mid x_{1}, x_{2}\right), \mathcal{Y}\right), p_{\text {ind }}\left(x_{1}, x_{2}\right)$ is a capacity-achieving distribution. Therefore, by [21. Theorem 4.5.1], for any $\left(x_{1}, x_{2}\right) \in \mathcal{S}_{\text {ind }}$,

$$
D\left(p\left(y \mid x_{1}, x_{2}\right) \| p_{\text {ind }}(y)\right) \leq I_{\text {ind }}\left(X_{1}, X_{2} ; Y\right),
$$

which further implies that for any distribution $p_{\text {dep }}\left(x_{1}, x_{2}\right)$ with support in $\mathcal{S}_{\text {ind }}$, we have

$$
\begin{aligned}
I_{\mathrm{dep}}( & \left.X_{1}, X_{2} ; Y\right)+D\left(p_{\text {dep }}(y) \| p_{\text {ind }}(y)\right) \\
= & H_{\text {dep }}(Y)-H_{\text {dep }}\left(Y \mid X_{1}, X_{2}\right)-H_{\text {dep }}(Y) \\
& -\sum_{y \in \mathcal{Y}} p_{\text {dep }}(y) \log p_{\text {ind }}(y) \\
= & \sum_{\left(x_{1}, x_{2}\right) \in \mathcal{S}_{\text {ind }}} p_{\text {dep }}\left(x_{1}, x_{2}\right) \sum_{y \in \mathcal{Y}} p\left(y \mid x_{1}, x_{2}\right) \log p\left(y \mid x_{1}, x_{2}\right) \\
& -\sum_{\left(x_{1}, x_{2}\right) \in \mathcal{S}_{\text {ind }}} p_{\text {dep }}\left(x_{1}, x_{2}\right) \sum_{y \in \mathcal{Y}} p\left(y \mid x_{1}, x_{2}\right) \log p_{\text {ind }}(y) \\
= & \sum_{\left(x_{1}, x_{2}\right) \in \mathcal{S}_{\text {ind }}} p_{\text {dep }}\left(x_{1}, x_{2}\right) D\left(p\left(y \mid x_{1}, x_{2}\right) \| p_{\text {ind }}(y)\right) \\
\leq & I_{\text {ind }}\left(X_{1}, X_{2} ; Y\right)
\end{aligned}
$$

Since in 76$), p_{\text {dep }}\left(x_{1}, x_{2}\right)$ is arbitrary, the $\operatorname{MAC}\left(\mathcal{X}_{1} \times\right.$ $\left.\mathcal{X}_{2}, p\left(y \mid x_{1}, x_{2}\right), \mathcal{Y}\right)$ is not in $\mathcal{C}^{*}$. This completes the proof.

\section{REFERENCES}

[1] M. Langberg and M. Effros, "On the capacity advantage of a single bit," in IEEE Globecom Workshops, 2016.

[2] P. Noorzad, M. Effros, and M. Langberg, "Can negligible cooperation increase capacity? The average-error case," in IEEE Int. Symp. Information Theory (ISIT), 2018.

[3] T. M. Cover and J. A. Thomas, Elements of Information Theory, 2nd ed. Wiley, 2006.

[4] F. M. J. Willems, "The discrete memoryless multiple access channel with partially cooperating encoders," IEEE Trans. Inf. Theory, vol. IT29, no. 3, pp. 441-445, 1983.

[5] P. Noorzad, M. Effros, and M. Langberg, "Can negligible rate increase network reliability?" IEEE Trans. Inf. Theory, vol. 64, no. 6, pp. 4282 4293, 2018.

[6] - "The unbounded benefit of encoder cooperation for the $k$-user MAC," IEEE Trans. Inf. Theory, vol. 64, no. 5, pp. 3655-3678, 2018.

[7] A. D. Sarwate and M. Gastpar, "Some observations on limited feedback for multiaccess channels," in IEEE Int. Symp. Information Theory (ISIT), 2009.

[8] A. P. Hekstra and F. M. J. Willems, "Dependence balance bounds for single-output two-way channels," IEEE Trans. Inf. Theory, vol. 35, no. 1, pp. 44-53, 1989.

[9] T. Ho, M. Effros, and S. Jalali, "On equivalence between network topologies," in Allerton Conf. Communication, Control, and Computing, 2010.

[10] S. Jalali, M. Effros, and T. Ho, "On the impact of a single edge on the network coding capacity," in Information Theory and Applications Workshop, 2011. 
[11] M. Langberg and M. Effros, "Network coding: Is zero error always possible?" in Allerton Conf. Communication, Control, and Computing, 2011.

[12] - "Source coding for dependent sources," in IEEE Information Theory Workshop, 2012.

[13] O. Kosut and J. Kliewer, "Strong converses are just edge removal properties," IEEE Trans. Inf. Theory, vol. 65, no. 6, pp. 3315-3339, 2019.

[14] P. Noorzad, "Network effects in small networks: A study of cooperation," Ph.D. dissertation, California Institute of Technology, June 2017.

[15] W. Gu, M. Effros, and M. Bakshi, "A continuity theory for lossless source coding over networks," in Proc. Allerton Conf. Communication, Control, and Computing, 2008.

[16] T. Chan and A. Grant, "On capacity regions of non-multicast networks," in Proc. IEEE Int. Symp. Information Theory, 2010.

[17] G. Dueck, "Maximal error capacity regions are smaller than average error capacity regions for multi-user channels," Probl. Contr. Inform. Theory, vol. 7, no. 1, pp. 11-19, 1978.

[18] S. L. Fong and V. Y. F. Tan, "A proof of the strong converse theorem in Gaussian multiple access channels," IEEE Trans. Inf. Theory, vol. 62 , no. 8, pp. 4376-4394, 2016.

[19] G. Dueck, "The strong converse of the coding theorem for the multipleaccess channel," Journal of Combinatorics, Information, and System Sciences, vol. 6, pp. 187-196, 1981.

[20] S. Saeedi Bidokhti and G. Kramer, "An application of a wringing lemma to the multiple access channel with cooperative encoders," in Iran Workshop on Communication and Information Theory (IWCIT), 2014.

[21] R. G. Gallager, Information Theory and Reliable Communication, 1st ed. Wiley, 1968

[22] T. M. Cover, A. El Gamal, and M. Salehi, "Multiple access channels with arbitrarily correlated sources," IEEE Trans. Inf. Theory, vol. IT-26, no. 6, pp. 648-657, 1980

[23] P. Noorzad, M. Effros, and M. Langberg, "Can negligible cooperation increase network reliability?" Sep. 2016. [Online]. Available: http: //arxiv.org/abs/1601.05769

[24] I. Csiszár and P. C. Shields, Information Theory and Statistics: A Tutorial. now Publishers, 2004

[25] M. R. Bloch, "Covert communication over noisy channels: A resolvability perspective," IEEE Trans. Inf. Theory, vol. 62, no. 5, pp. 2334-2354, 2016

[26] L. Wang, G. W. Wornell, and L. Zheng, "Fundamental limits of communication with low probability of detection," IEEE Trans. Inf. Theory, vol. 62, no. 6, pp. 3493-3503, 2016.

[27] C. C. Pugh, Real Mathematical Analysis, 1st ed. Springer, 2001.

[28] D. Bertsimas and J. N. Tsitsiklis, Introduction to Linear Optimization. Athena Scientific, 1997

Parham Noorzad (S'11-M'17) received the B.Sc. degree in electrica engineering from the University of Tehran in 2012 and the M.Sc. and Ph.D degrees in electrical engineering from the California Institute of Technology in 2013 and 2017, respectively. His research interests include machine learning, wireless communications, and information theory.

Michael Langberg (M'07-SM'15) received his B.Sc. in mathematics an computer science from Tel-Aviv University in 1996, and his M.Sc. and Ph.D. in computer science from the Weizmann Institute of Science in 1998 and 2003 respectively. Between 2003 and 2006, he was a postdoctoral scholar in the Electrical Engineering and Computer Science departments at the California Institute of Technology, and between 2007 and 2012 he was in the Department of Mathematics and Computer Science at The Open University of Israel. Prof. Langberg is currently a professor in the Department of Electrical Engineering at the University at Buffalo.
Prof. Langberg's research addresses the mathematical foundations of information, and in particular the design and analysis of efficient and reliable algorithms for the communication, management, and storage of information. Major topics of study include the fundamental algorithmic and combinatorial challenges that arise in the study of point-to-point communication, network communication, data storage, and succinct data representation. Prof. Langberg was an Associate Editor for the IEEE Transactions on Information Theory during the years 2012-2015 and the Editor of the IEEE Information Theory Society Newsletter during the years 2015-2018.

Michelle Effros (S'93-M'95-SM'03-F'09) received the B.S. (Hons.), M.S., and $\mathrm{Ph} . \mathrm{D}$. degrees in electrical engineering from Stanford University, in 1989, 1990, and 1994, respectively.

She joined the faculty at the California Institute of Technology in 1994, where she is currently the George Van Osdol Professor of Electrical Engineering. Her research interests include information theory, network coding, data compression, and communications. She is a member of Tau Beta Pi, Phi Beta Kappa, and Sigma Xi. She was a member of the Advisory Committee and the Committee of Visitors for the Computer and Information Science and Engineering (CISE) Directorate at the National Science Foundation from 2009 to 2012 and in 2014, respectively. She received the Stanford's Frederick Emmons Terman Engineering Scholastic Award (for excellence in engineering) in 1989, the Hughes Masters Full-Study Fellowship in 1989, the National Science Foundation Graduate Fellowship in 1990, the AT\&T Ph.D. Scholarship in 1993, the NSF CAREER Award in 1995, the Charles Lee Powell Foundation Award in 1997, the Richard Feynman-Hughes Fellowship in 1997, and an Okawa Research Grant in 2000. She was cited by Technology Review as one of the world's top young innovators in 2002 . She and her coauthors received the Communications Society and Information Theory Society Joint Paper Award in 2009. She served as an Editor of the IEEE Information Theory Society Newsletter from 1995 to 1998 and as a member of the Board of Governors of the IEEE Information Theory Society from 1998 to 2003 and from 2008 to 2017, serving in the role of President of the Information Theory Society in 2015. She served on the IEEE Signal Processing Society Image and Multi-Dimensional Signal Processing (IMDSP) Technical Committee from 2001 to 2007 and on ISAT from 2006 to 2009. She served as an Associate Editor for the 2006 joint Special Issue on Networking and Information Theory in the IEEE Transactions on Information Theory and the IEEE Transactions on Networking/ACM Transactions on Networking and as an Associate Editor for Source Coding for the IEEE Transactions on Information Theory from 2004 to 2007. She has served on numerous technical program committees and review boards, including serving as the General Co-Chair for the 2009 Network Coding Workshop and the Technical Program Committee Co-Chair for the 2012 IEEE International Symposium on Information Theory. 